\title{
Recent Advancements in Rayleigh Scattering-Based Distributed Fiber Sensors
}

\author{
Xiaoyi Bao $\mathbb{D}^{1,2}$ and Yuan Wang $\mathbb{D}^{1}$ \\ ${ }^{1}$ School of Electrical Engineering and Computer Science, University of Ottawa, Ontario, Canada K1N 6N5 \\ ${ }^{2}$ Department of Physics, University of Ottawa, 25 Templeton Street, Ottawa, Ontario, Canada K1N 6 N5
}

Correspondence should be addressed to Xiaoyi Bao; xbao@uottawa.ca

Received 6 November 2020; Accepted 18 February 2021; Published 11 March 2021

Copyright (c) 2021 Xiaoyi Bao and Yuan Wang. Exclusive Licensee Beijing Institute of Aerospace Control Devices. Distributed under a Creative Commons Attribution License (CC BY 4.0).

\begin{abstract}
Recently, Rayleigh scattering-based distributed fiber sensors have been widely used for measurement of static and dynamic phenomena such as temperature change, dynamic strain, and sound waves. In this review paper, several sensing systems including traditional Rayleigh optical time domain reflectometry (OTDR), $\Phi$-OTDR, chirped pulse $\Phi$-OTDR, and optical frequency domain reflectometry (OFDR) are introduced for their working principles and recent progress with different instrumentations for various applications. Beyond the sensing technology and instrumentation, we also discuss new types of fiber sensors, such as ultraweak fiber Bragg gratings and random fiber gratings for distributed sensing and their interrogators. Ultimately, the limitations of Rayleigh-based distributed sensing systems are discussed.
\end{abstract}

\section{Introduction}

Infrastructure, such as buildings, roads, and highways, are critical to the day to day lives of people around the world. These structures provide human beings with a better and more convenient life. However, these structures have their lifetimes and could deteriorate. The deterioration is commonly the result of aging of materials, overloading, and lack of sufficient maintenance. To ensure structural safety and prevent disasters in advance, civil structures have to be equipped with structural health monitoring (SHM). SHM provides an effective tool to assess structural health conditions and helps to avoid losses for users due to structural failure. An effective SHM system should be real-time monitoring and be able to detect various locations at the same time. Distributed fiber optic sensor technology is one of the most promising candidates among the numerous sensor technologies that are adopted for SHM [1]. Because it can determine the value of an object variable in a continuous way, distributed sensors allow evaluation of the system without causing damage, saving both money and time in product evaluation and infrastructure monitoring, especially optical fibers, and have advantages of being lightweight and immune to electromagnetic interference, which increase the distributed sensing application areas for SHM.
The basic principle of distributed fiber optic sensors is optical time domain reflectometry (OTDR) which was firstly introduced to monitor fiber attenuation [2]. In the OTDR technique, a narrow optical pulse is used as the probe signal to interrogate the optical fiber under test, then a Rayleigh backscattering signal from the fiber material is detected, and the external perturbation-induced changes can be detected due to the amplitude of Rayleigh patter changes. The location is mapped through flight time of a pulse of light traveling to and from the pattern changed location. For this reason, the spatial resolution is determined by the duration of the optical pulse. The spatial resolution can be improved as the pulses are shortened at the expense of the broadened bandwidth, reduced interaction length, and increased noise level and consequently in the reduction of dynamical range (a well-known trade-off between dynamical range and spatial resolution). As a result, the resolution of measured parameters, such as temperature, strain, vibration, and acoustic wave, will be compromised. OTDR-based sensors often use direct detection to measure local intensity changes. A few techniques have been introduced to enhance the weak Rayleigh signal, such as ultraweak FBG arrays [3] and random fiber grating arrays [4]; they have reflection of around $-30 \mathrm{~dB}$ which is stronger than the Rayleigh signal. The enhanced period and irregular pattern can be deployed by 


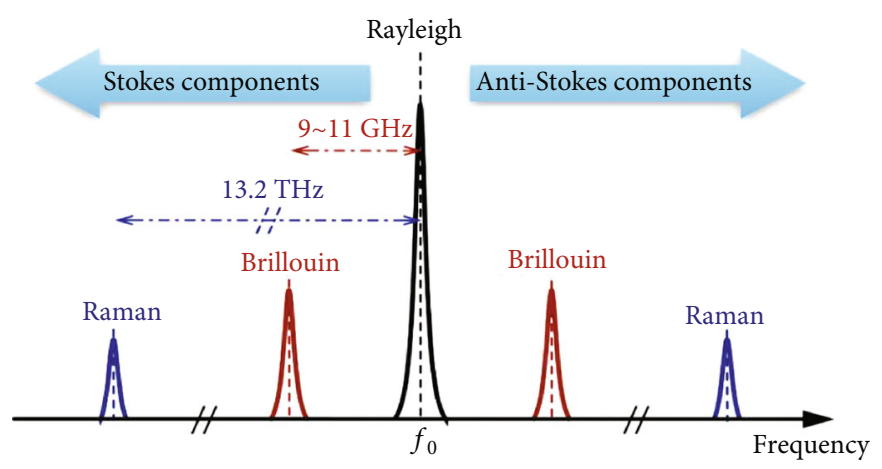

Figure 1: The spectrum of backscattered light in the optical fibers.

coherent phase OTDR to recover temperature-, strain-, or vibration-induced phase changes at higher precision than telecom fiber-based OTDR sensors [5].

Another Rayleigh scattering-based approach is the optical frequency-domain reflectometry (OFDR) with the frequency scanning, in which the probe signal is a continuous frequency modulated optical wave instead of a pulsed signal as in OTDR [6]. In contrast to OTDR, the OFDR systems have relatively higher power per frequency components, so a more dynamic range is then obtained. The spatial resolution is determined by the tuning range of the laser, which can be one millimeter for sensing of temperature or strain; the sensing length is limited by the coherent length of the laser to tens of meters, typically for a commercial system. The demodulation scheme of OFDR is interferometric, so it measures relative phase difference associated with temperature or strain variations.

This paper reviews the recent advancement in Rayleigh scattering-based OTDR, phase-sensitive OTDR using standard single mode fiber, ultraweak FBGs, and random fiber grating array for distributed fiber sensors in temperature, strain, and vibration measurements, as well as phase OTDR application to measure laser linewidth and phase noises, chirped pulse-based phase-sensitive OTDR technology, and challenges for Rayleigh-based OTDR and OFDR sensors.

The layout of this paper is arranged as follows: the introduction to distributed sensing is presented in Section 1, the theory and working principle of spontaneous Rayleigh scattering and their mechanisms for measuring strain and temperature are presented in Section 2, the Rayleigh scatteringbased OTDR and OFDR system performances are detailed in Sections 3 and 4, Section 5 will summarize application needs for distributed sensors, and Section 6 is the conclusion.

\section{Spontaneous Scattering in Optical Fibers}

When a light wave with wavelength of $\lambda_{0}$ propagates in a medium, it interacts with the atoms and molecules in that medium. When $\lambda_{0}$ is far from a medium resonance, the electric field induces a time-dependent polarization dipole. The induced dipole generates a secondary electromagnetic wave, which is light scattering. Because the distances between scattering centers (particles) are smaller than the wavelength of light in optical fibers, the secondary light waves and original waves are coherent. Hence, the resulting intensity is the addition of the scattered fields.

When the medium is perfectly homogeneous, the phase relationship of the emitted waves only allows the forward scattered beam. The medium can then be considered as continuous, and the scattered lights are coherently added; there is no backward scattering loss. However, optical fiber is an inhomogeneous medium; scattering arises from microscopic or macroscopic variations in density and composition. The random order of the molecules and the presence of dopants cause localized variations in density, and therefore, the refractive index varies along the fiber. They are caused by small particle sizes $(\ll \lambda)$, such as electrons, atoms, molecules, or nanoparticles. The scattered light maintains the same energy with the changed direction. Scattering from spherical particles with dimension $>\lambda / 10$ is called Mie scattering [7]. The strength of Mie scattering does not have strong dependence on the pump wavelength. Rayleigh scattering causes attenuation of the forward-propagating signal (and creation of a backward-propagating wave) that is proportional to $1 / \lambda^{4}$. Rayleigh scattering is a linear scattering process, which means scattered power is proportional to the incident power, and no energy is transferred to the glass in Rayleigh scattering; therefore, there is no change in frequency of the scattered light comparing with the wavelength of the incident light, the so called elastic scattering.

In Figure 1, the backward scattering spectrum from materials includes Rayleigh scattering at the incident frequency, and two lines appearing on red-shifted and blueshifted sidebands of the Rayleigh peak are the Brillouin lines. These two peaks present the scattering of sound waves moving in opposite directions in the Brillouin scattering process. The left peak with a downshifted frequency is called the Stokes wave, while the right one with an upshifted frequency is called the anti-Stokes wave. Raman lines are contributed by the interaction of the incident photon with the molecular vibration state. The optical fiber with broad vibrational spectrum gives broad Raman spectrum. Both Brillouin and Raman scatterings are inelastic scatterings because they are associated with certain frequency shifts. The last mechanism that can be observed is the Rayleigh wing scattering attributed to fluctuations in the orientation of anisotropic molecules. 
Raman spectra in crystals usually contain many sharp bands where separations between bands, corresponding to the electronic vibrations, and each bandwidth result from molecular rotation or reorientation excitations. In optical fibers, Raman scattering is presented as a broadband spectrum.

2.1. Rayleigh Scattering in an Optical Fiber. Rayleigh scattering is caused by inhomogeneity of the fiber medium. The random ordering of the molecules causes localized variations of density and therefore fluctuations of the refractive index. Rayleigh scattering is an elastic scattering, and the frequency of scattered light is the same as the incident light. Rayleigh scattering is the most important factor to determine the transmission loss of an optical fiber [8], and it is caused by the density fluctuations frozen into the fused silica during the manufacturing process. It results in the refractive index change along the fiber. The intrinsic loss coefficient of the optical fiber due to Rayleigh scattering is expressed as [9]

$$
\alpha_{R}=\frac{A}{\lambda^{4}}
$$

where the constant $A \approx 0.7-0.9\left(\mathrm{~dB} \cdot \mathrm{km}^{-1} \cdot \mu \mathrm{m}^{-4}\right)$.

Rayleigh scattering and material absorption are two mechanisms for the fiber loss. The total attenuation coefficient is denoted as $\alpha$ (with the units of $\mathrm{km}^{-1}$ ). In the wavelength of $1550 \mathrm{~nm}$ which is commonly used in the optical communication, the attenuation coefficient is about $0.2 \mathrm{~dB} / \mathrm{km}$ in the standard single mode fiber (SMF). Considering the scattering coefficient in the range $0.12-0.16 \mathrm{~dB} / \mathrm{km}$, Rayleigh scattering is the dominant loss near that wavelength. This provides a way to estimate the fiber attenuation by monitoring the Rayleigh backscattering power.

Due to the attenuation loss, the input optical power will decay exponentially as a function of the propagating distance $z$ as shown in the following equation:

$$
P(z)=P_{0} e^{-\alpha z}
$$

where $P_{0}$ is the power of the incident light. $P(z)$ is the transmitted power at position $z$ which is between $z=0$ and total length $L$. In Equation (2), the unit of $\alpha$ is $\mathrm{km}^{-1}$ and $\alpha_{\mathrm{dB}} \approx$ $4.34 \alpha$. The attenuation coefficient $\alpha$ can be expressed as $\mathrm{dB} / \mathrm{km}$ in the telecom industry and research field. Then, the transmitted power can be written as

$$
P(z)=P_{0} 10^{-\left(\alpha_{d B} z / 10\right)}
$$

In summary, Rayleigh scattering is an elastic scattering where the scattered light frequency is the same as that of the incident light. In the small spatial scale (tens of molecular sizes), one would observe fluctuations in local temperature or strain values under incident light on a material; this EM field $E$ would reorient the originally incoherent random fluctuating molecular clouds to have a tendency to respond collectively in the same way on a small spatial scale covering the wavelength of the EM field. Such collective tendency to respond to an EM field would result in macroscopic polariza- tion $P=\varepsilon_{0} \chi E$, which is proportional to the external electric field $E$. The parameter $\chi$ is a randomly fluctuating term in the medium with $\Delta \varepsilon(t, z)$, which is the fundamental reason that Rayleigh scattering can be used to sense vibration, temperature, and strain. This fluctuating dielectric parameter $\Delta \varepsilon$ gives a fluctuating polarization-induced light emission in all directions. Some of the scattered Rayleigh lights are recaptured by the single mode or multimode fiber in the backward direction. This backward propagating Rayleigh scattered light has a time delay that can be used to refer the spatial location of the scattering. Hence, Rayleigh optical time domain reflectometry (OTDR) can locate the fiber condition with or without temperature or strain (static or dynamic) disturbance.

\section{Distributed Fiber Sensors Based on Rayleigh Scattering}

There are great efforts in Rayleigh scattering-based distributed sensors in last decade, especially in enhanced scattering by random fiber grating and weak FBGs. The applications of the distributed sensors based on Rayleigh scattering have been extended to sense laser dynamics, which includes the laser noises and the laser linewidth measurement.

3.1. Rayleigh OTDR. When a light wave prorogues along an optical fiber, the light power will decrease continuously mostly due to Rayleigh scattering which results from the inhomogeneity of the refractive index during the fiber fabrication process. The random localized fluctuation of the molecule position in the optical fiber can be regarded as small scattering centers with a dimension much smaller than the optical wavelength.

If a laser pulse with peak power $P_{0}$ and pulse duration $T$ is injected into one end of the fiber, the Rayleigh scattering signal will drop by $d P_{R}$ over the fiber length $d_{z}$ [10]:

$$
\frac{d P_{R}}{d z}=C_{b} \alpha_{R} P_{0} e^{-2 \alpha z},
$$

where $\alpha$ is the fiber attenuation and $z=v_{g} t / 2 n$ with changes from 0 to $L$. $C_{b}$ is the ratio of Rayleigh scattering captured by optical fibers in the backward direction, which is expressed by

$$
C_{b}=\left(\frac{\mathrm{NA}}{n_{1}}\right)^{2} \times \frac{1}{m}=\left(\frac{\sqrt{n_{1}^{2}-n_{2}^{2}}}{n_{1}}\right)^{2} \times \frac{1}{m} .
$$

$\mathrm{NA}$ is the numerical aperture and $n_{1}$ and $n_{2}$ are the refractive indexes of the core and the cladding, respectively. Factor $m$ varies with the refractive index profile of the fiber.

For a Gaussian beam waist of $\omega(\lambda, Z)$, the recapture coefficient is expressed by [10]

$$
C_{b}(\lambda, Z)=\frac{3}{2}\left(\frac{\lambda}{2 \pi n_{1} \omega(\lambda, z)}\right)^{2} .
$$




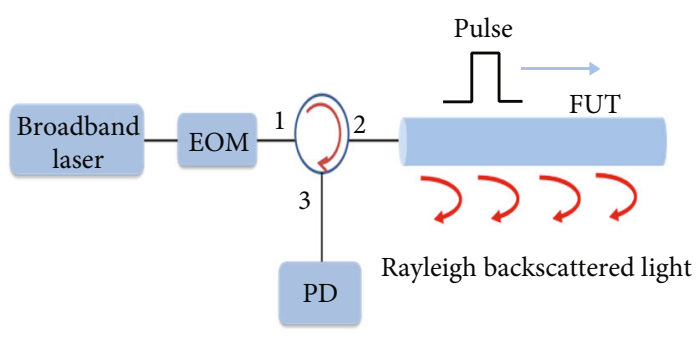

(a)

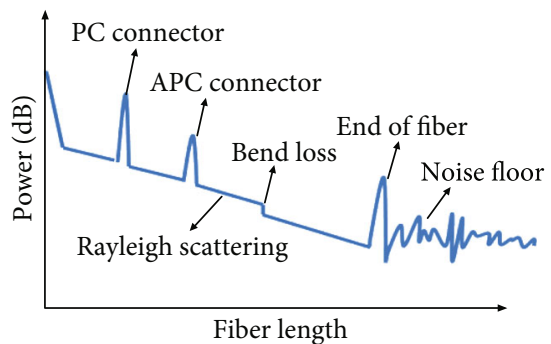

(b)

FIGURE 2: (a) Experimental setup of typical OTDR system. (b) Reflected Rayleigh traces.

The Rayleigh signal power can be obtained from solving Equation (4) and expressed as

$$
P_{R}(z)=\frac{1}{2} \alpha_{R} P_{0} V_{g} T C_{b}(z) e^{-2 \alpha z}
$$

Rayleigh OTDR was developed for fault detection in the telecommunication fiber. It is based on inhomogeneityinduced Rayleigh scattering loss at one participle wavelength along the optical fibers by measuring power change in Equation (4). A simplified OTDR setup is shown in Figure 2(a). The laser source used in an OTDR system is a broadband light source which is modulated by an EOM to generate optical pulses. Then, the optical pulses are sent into the sensing fiber through a circulator, and the Rayleigh backscattered light is detected by a photodetector (PD). The detected optical power decreases exponentially with respect to the distance along the fiber and shows abrupt peaks and dips at the location of connectors and bends and breaks due to Fresnel reflections, which is shown in Figure 2(b).

The vertical scale represents the reflected signal level on a logarithmic scale, while the horizontal scale corresponds to the distance along the fiber or device under test. Since OTDR can only measure the time delay directly, such a time delay can be converted to distance by $z=V_{g}(z) t / 2$. $V g(z)=c / n_{g}(z)$ is the group velocity of the light propagating in the optical fiber.

The spatial resolution $\Delta z_{\min }$ of an OTDR distributed sensor represents the ability to locate fault point or unusual temperature or strain section. It can be expressed as $\Delta z_{\min }=c \tau / 2 n$. Here, $\tau$ is the pulse width of the incident light, $n$ is the refractive index of the fiber core, and $c$ is the speed of light in vacuum. The dynamic range is defined as the difference between the initial backscattered power level and the noise level of a measurement time. The way to increase the system's dynamic range is either to enhance the backscattered power or to decrease the noise level. From Equation (6), the backscattered power is proportional to the product of the pulse width and input power. However, in the OTDR system, the pulse with high peak power is not desired because it will cause the nonlinear effects in the fiber. So the dynamic range mainly depends on the pulse width. Increasing the dynamic range requires the longer pulses which would decrease the spatial resolution. This trade-off between the dynamic range and spatial resolution represents the fundamental limit of the OTDR system. Thus, designing the system needs to consider both parameters tailored to different applications.

One source of loss is microbending loss, and a distributed sensor system to measure lateral pressure on a fiber has been developed [11] that places a fiber inside a spiral sheath that induces microbending in the fiber when a lateral force is applied. The original idea was developed for mechanical sensing based on microbending-induced loss from small and sharp bends on fibers [12], which increases local attenuation along the single mode fiber. The extra loss is detected through standard OTDR techniques.

Distributed temperature sensing using the conventional OTDR system has been investigated by monitoring the temperature-induced amplitude changes of the Rayleigh backscattering light. This kind of temperature sensor has been accomplished in a special liquid-core fiber with an accuracy of $\pm 1^{\circ} \mathrm{C}$ and a spatial resolution of a few meters [13]. The liquid-core fiber is fabricated by a glass tube (the cladding) filled with a liquid with higher refractive index to form the fiber core. The temperature dependence of the scattering loss due to thermal disturbance of the liquid has much stronger temperature dependence than that in the telecom fiber. However, the requirement of the liquid core in the fiber has limitation in the field, where large temperatures ranging from $-30^{\circ} \mathrm{C}$ to $+40^{\circ} \mathrm{C}$ could occur. Another temperature sensor in conventional OTDR is based on the temperature-dependent attenuation coefficient of doped glass fibers. Since the absorption bands of the dopant changes with temperature, the temperature changes can be determined by measuring the loss near the edge of an absorption band. Based on this principle, distributed temperature measurement using $\mathrm{Nd}$-doped fiber shows $2^{\circ} \mathrm{C}$ temperature accuracy and $15 \mathrm{~m}$ spatial resolution over $140 \mathrm{~m}$ sensing fiber length [14]. Using Ho-doped fiber, these figures were improved to $1^{\circ} \mathrm{C}$ and $3.5 \mathrm{~m}[15]$.

\subsection{Phase-Sensitive OTDR ( $\Phi-O T D R)$}

3.2.1. Principle of $\Phi-O T D R$. The Rayleigh OTDR in Section 3.1 used a broadband light source, so that the coherent length is less than the pulse width, and the detected signal represents intensity addition instead of field addition with interference. $\Phi$-OTDR configuration used a narrow linewidth laser whose coherent length is much longer than the fiber length. The detected signal represents field addition, which is the 


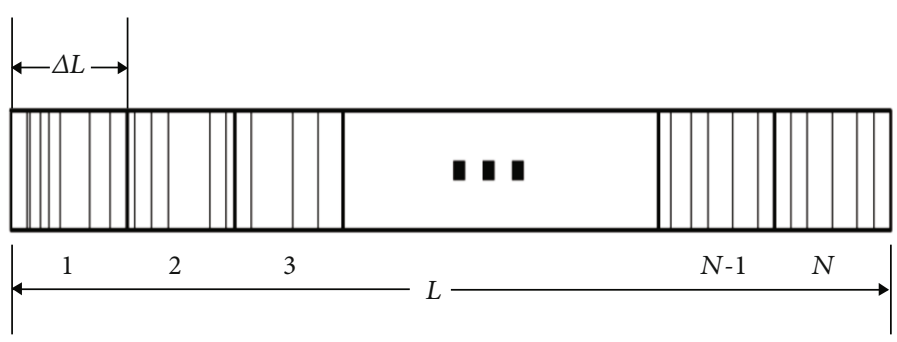

(a)

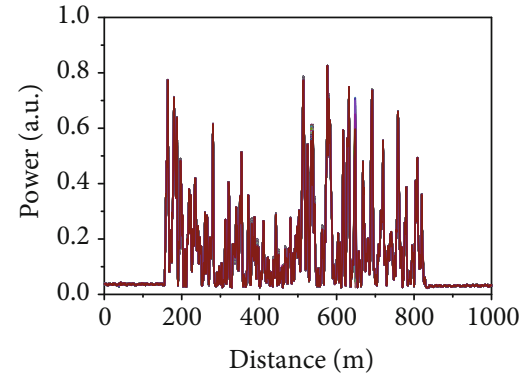

(b)

Figure 3: (a) The schematic of simulation model of $\Phi$-OTDR system. (b) Reflected Rayleigh traces.

summing up of Rayleigh scattering of many scattering centers within the pulse width. These scattering centers are equivalent as multiple reflectors with weak reflectivity. When the fiber is under the disturbance of the temperature, strain, or vibration change, the optical path length between the scattering centers will change accordingly. This induced interference pattern changes locally with time, which can be demodulated by phase or intensity. In order to understand the principle of $\Phi$-OTDR, a simplified simulation model has been developed, and the schematic is shown in Figure 3(a) [16].

The fiber can be divided into $N$ small sections and the length for each section is $\Delta L=L / N$. $\Delta L$ is equivalent to the same length as the pulse width in the simulation. In each section of the fiber, there are $M$ scattering centers which are randomly located with uniform distribution. So the electric field of Rayleigh backscattered light can be written as

$$
E_{b s}^{i}=E_{0} \exp \left(-2 \alpha L_{i}\right) \sum_{k=1}^{M} r_{k}^{i} \exp \left(j \varphi_{k}\right)
$$

where $\alpha$ is the attenuation coefficient of the optical fiber, $L_{i}$ is the length of the $i^{\text {th }}$ section of the fiber, and $L_{i}=i \Delta L . r_{k}$ and $\varphi_{k}$ are the scattering coefficient and phase of the $k^{\text {th }}$ scattering center, respectively. In $\Phi$-OTDR, backscattered light within the pulse width interferes with each other and forms a random interference pattern. Here, we can assume the scattering coefficient for every scattering center is the same, denoted by $r$. Then, intensity of the detected signal can be expressed as [17]

$$
\begin{aligned}
I_{b s}^{i}= & \left|E_{b s}^{i}\right|^{2}=\sum_{k=1}^{M-1} \sum_{k^{\prime}=k+1}^{M} E_{0}{ }^{2} r \exp \left(-4 \alpha L_{i}\right) \\
& +2 E_{0}^{2} r^{2} \sum_{k=1}^{M-1} \sum_{k^{\prime}=k+1}^{M} \cos \left(\varphi_{k^{\prime}}-\varphi_{k}\right) .
\end{aligned}
$$

The first term in the detected signal is the DC component which can be removed by an AC coupled detection. The second term is the sum of multiple interference signals, and its intensity depends on the combination of the phase difference within the pulse width. From Equation (8), the intensity of the detected signal gives the relative phases of reflected light from different scattering centers within the pulse width. Since the scattering centers are randomly distributed along the fiber, the $\Phi$-OTDR traces typically have random oscillating features as shown in Figure 3(b). This random pattern remains the same over time if the scattering centers do not suffer from any changes. If there is any disturbance at one certain location, the relative phases of backscattered light are changed, and $\Phi$-OTDR traces will vary at that disturbance location. Thus, the disturbance location can be obtained by tracking the difference between unperturbed and perturbed signals via trace subtraction or correlation. In the case of vibrations, the $\Phi$-OTDR traces can show local variations synchronized with the vibration frequency. Trace superposition of $\Phi$-OTDR around the vibration location is represented by Figure 4(a) (100 traces). The intensities at other locations remained the same for every trace except around $650 \mathrm{~m}$ where there is a vibration event. After subtracting adjacent traces, the vibration location is clearly shown in Figure 4(b). The vibration frequency can be obtained by performing the fast Fourier transform (FFT) at the vibration location along the sequence traces.

\subsubsection{Detection Methods and Noise in $\Phi-O T D R$}

(1) Noise Sources. There are several noise sources in the direct detection: (i) laser phase noise, (ii) laser frequency drift, and (iii) intraband noise caused by the finite extinction ratio of the optical pulse. The laser phase noise is determined by the linewidth of the laser which could affect the performance of $\Phi$-OTDR for vibration sensing [18]. In order to reduce the fluctuation of phases, the coherence length of the laser should be longer than the pulse width. The laser frequency drift would also cause the fluctuation of $\Phi$-OTDR traces since the interference patterns would change in different laser frequencies [19]. Therefore, in order to get stable traces in $\Phi$-OTDR, the system requires the low phase noise and small frequency drift laser with stable frequency over the measurement time. The active compensation method that utilizes the laser frequency sweeping and cross-correlation calculation has been proposed to suppress the trace distortion [20]. In addition, the fluctuation of traces from the EOM bias drift during the measurement can also be an issue, because it induces distortion of the extinction ratio (ER) of the optical pulse. This will result in a CW component between pulses and then generate a $\mathrm{CW}$ backscattered light that would 


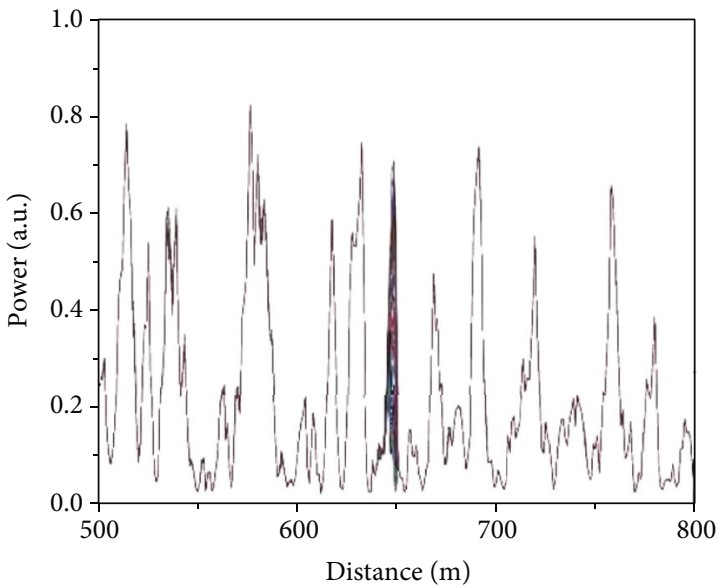

(a)

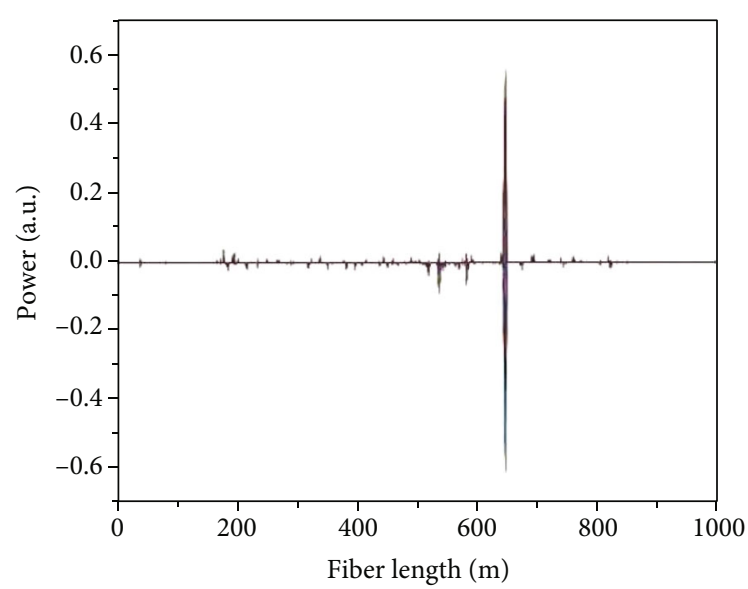

(b)

FIgURE 4: (a) Experimental $\Phi$-OTDR traces with the vibration at $650 \mathrm{~m}$; (b) the vibration location after trace subtraction.

decrease the SNR. The CW noises can accumulate with the fiber length as a coherent Rayleigh signal; it could limit $\Phi$-OTDR in long range sensing and reduce spatial resolution for different applications. The EOM feedback control in $\Phi$-OTDR is introduced for solving the bias drift problem [21].

(2) Direct Detection. The direct detection scheme presents the low complexity for the signal being directly detected in the time domain. It is suitable for the short range sensing measurements where the backscattering light is relatively strong.

(3) Coherent Detection. Due to the weak Rayleigh backscattering in the optical fiber, the use of coherent detection can greatly increase the SNR and sensitivity of a detected signal. The principle of coherent detection consists of mixing the optical signal with a local oscillator (LO) before it reaches the photodetector.

The light coming from a laser with a frequency $f_{0}$ is split by an optical coupler into two arms. One arm is used as LO, and the other is used for generating the backscattered signal as shown in Figure 5. In coherent detection, the frequencies of optical pulses are usually shifted $\Delta f$ (usually few hundred $\mathrm{MHz}$ ) before sending into the fiber to overcome the low frequency noise from the environment. In this case, the detected signal becomes the interference between the LO and the Rayleigh backscattered light. Because the optical amplitude of the LO signal is typically much higher than that of the backscattered signal, the intensity of the interference signal in this case will be higher than that in the direct detection. If the optical amplitude of LO is high enough, the SNR of coherent detection can be reached to the shot noise limit even for weak signals, while the direct detection cannot achieve this. Therefore, the coherent detection is more suitable for weak signal detection compared to direct detection.

The main disadvantages of using coherent detection are higher noises and instability of $\Phi$-OTDR traces. Besides the noise occurring in the direct detection, the coherent detec- tion is much more sensitive to the phase noise and strongly dependent on the state of polarizations of two arms. In the coherent detection, the polarization mismatch between two arms can deteriorate the interference signal at some positions. By using the polarization maintaining fiber (PMF), as well as the polarization maintaining components, the polarization-dependent problem has been solved [22]. But PMFs are expensive and have relatively high loss which limits the sensing range when compared to the usage of SMF. The polarization diversity detection could also solve this problem [17].

(4) Polarization Diversity Detection. The $\Phi$-OTDR system operates based on coherent Rayleigh scattering light by utilizing a high-coherence laser with narrow linewidth. The detected signals are induced by the interference between multiple Rayleigh scattering centers within a pulse width. External perturbations including acoustic waves or vibration applied on the fiber would modulate the effective refractive index of the guided optical mode, causing a phase shift of reflected light as well as the change in the intensity of detected signals. The $\Phi$-OTDR was firstly realized using a direct detection scheme as an intrusion sensor with a simple setup; however, a relatively low SNR was obtained [23, 24]. By using the postsignal processing methods such as moving average and wavelet denoising, the $\Phi$-OTDR with high sensitivity and SNR has been demonstrated, which allows the acoustic wave and vibration spectrum to be measured in a distributed fashion [22].

The performance improvement in an $\Phi$-OTDR system was achieved by using a heterodyne coherent detection technique [25]. However, the coherent detection is susceptible to the polarization mismatch between the measured and reference signals, and hence, the measurement accuracy can be greatly affected by the polarization-induced fading phenomenon [26]. The polarization mismatch would decrease the SNR of $\Phi$-OTDR time-domain traces and cause the low visibility of interference signals. Unreliable vibration 


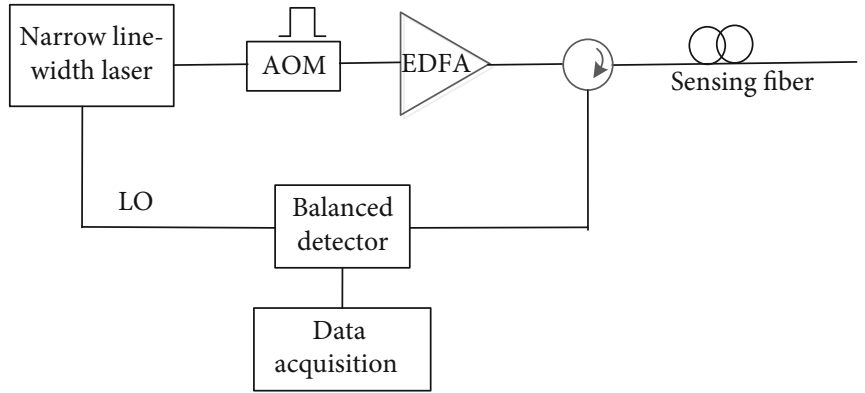

FIGURE 5: Schematic diagram of a $\Phi$-OTDR system based on coherent detection.

measurement results may occur at polarization mismatched locations because of these disturbance-insensitive regions.

For single-point sensing applications, a polarization controller is introduced in the reference arm to align the polarization at certain locations. For distributed sensing applications, since the state of polarization of Rayleigh backscattered light changes randomly along the lowbirefringence optical fiber, it is impossible for the PC to keep polarization alignment for the backscattered signals at each location along the sensing fiber. Thus, the probability of finding the useful polarization matched locations could be significantly reduced. This problem becomes even more serious in multiple vibration event detection. It could be a major obstacle in the real field such as intrusion detection, leakage detection of pipeline monitoring, internal crack of the bridge, and oil-well condition monitoring. To mitigate the polarization fading effect, one approach is to utilize the highbirefringence polarization-maintaining fiber configurations [27]. In addition, the polarization diversity (PD) method [28] provides an alternative solution for $\Phi$-OTDR to reduce the polarization fading for the telecom fiber by separating the backscattering signals into two orthogonal polarization states through a polarization beam splitter (PBS) and then combine the two separated signals so that the detected signals are insensitive to the SOP in fibers.

$\Phi$-OTDRs based on the PD method compensate the position-dependent polarization fading occurring in the coherent detection. A real distributed vibration sensing for multievent detections can thus be achieved at each position along the sensing fiber with more reliable vibration measurement results. The experiment shows that the SNR of two vibration signals are simultaneously enhanced by $10.9 \mathrm{~dB}$ and $8.65 \mathrm{~dB}$ compared to the coherent detection scheme [17]. This technique eliminates the polarization mismatch between the sensing arm and the reference arm at any given location of the sensing fiber, which makes it more suitable for the multievent sensing applications.

3.3. $\Phi-O T D R$ for Quantitative Temperature and Strain Measurement. Koyamada et al. [29] proposed and demonstrated the Rayleigh scattering-based OTDR for distributed temperature and strain measurement. They used a laser with precisely controlled optical frequency combining the coherent detection for $\Phi$-OTDR trace collection. The Rayleigh scattering is formed by many randomly spaced scattering centers at nanometer separation, and the sum of the scatter- ing centers can be added coherently as their spacing is much smaller than the optical wavelength. This response is similar to FBG, except FBG has equal spacing, while in Rayleigh scattering, the spacing varies randomly at a range of a few $\mathrm{nm}$ to hundreds of $\mathrm{nm}$ in the spatial distance. Just like FBG has spectral response with a profile, the wavelength shift of the Rayleigh scattering pattern is linearly proportional to the temperature and strain with the following relation [29]:

$$
\begin{aligned}
& \frac{\Delta v}{v_{0}} \approx-0.78 \times \Delta \varepsilon, \\
& \frac{\Delta v}{v_{0}} \approx-\left(6.82 \times 10^{-6}\right) \times \Delta T,
\end{aligned}
$$

where $v_{0}$ is the nominal laser frequency, $\Delta v$ is the laser frequency change relative to reference temperature or strain associated laser frequency, and $\Delta \varepsilon$ is the changed strain relative to the reference strain $\varepsilon_{0}$. The same principle applies to the temperature change $\Delta T$.

The spectral pattern of the Rayleigh scattering also changes with temperature or strain linearly. The slope of this pattern is a constant for the telecom fiber. If one can record the laser frequency (wavelength) at one temperature or strain as reference and calibrate a range of the temperature and strain for that type of the fiber, then the temperature and strain can be measured in a distributed fashion, if a pulse laser is used. The pulse width in the time domain can be transferred to the spatial domain based on $z=c t / 2 n(z)$. If the laser frequency can be measured accurately, the measured temperature accuracy can be as high as $0.01^{\circ} \mathrm{C}$ for the longterm laser frequency stability $<5 \mathrm{MHz}$ over the optical frequency of $10^{14} \mathrm{~Hz}$ with linewidth of $3 \mathrm{MHz}$, and the pulse width is $10 \mathrm{~ns}$, which is equivalent to $1 \mathrm{~m}$ spatial resolution.

$\Phi$-OTDR detects the relative wavelength shifts for the temperature or strain change over the reference temperature or strain. This measurement allows high temperature resolution, because the optical frequency change can be converted to a microwave regime which can be measured at much higher accuracy than the optical frequency measurement, which gives high temperature precision. Note the laser frequency drift should be much smaller than the Rayleigh pattern change due to the strain or temperature. The accuracy of microwave frequency measurement between reference and tested temperature and strain can be measured at the $\mathrm{MHz}$ level, which is proportional to the strain and 
temperature accuracy according to Equation (3.5). The reference temperature and strain should be updated every time before the real measurement to reduce the laser frequency drift-induced error.

The principle for the $\Phi$-OTDR to measure distributed temperature or strain is to record Rayleigh pattern change at one temperature or strain at one specific wavelength at every fiber location using an optical pulse from a narrow linewidth laser, often the linewidth of $\mathrm{kHz}$. When the temperature or strain is changed at certain fiber locations, then the Rayleigh pattern in that location will be changed accordingly. By scanning the laser wavelength until the same Rayleigh pattern is recovered at every fiber location, one can get temperature or strain information using Equation (3.5). The spatial resolution is equivalent to the optical pulse length, due to weak Rayleigh scattering in the optical fiber within spatial resolution; the optical spatial resolution is often limited to $1 \mathrm{~m}$.

The same principle is applied to the phase recovered based $\Phi$-OTDR for strain measurement [30], based on interferometers for phase measurement rather than frequency measurement. Phase measurement lowers the requirement of the expansive frequency stabilized laser; any phase locked laser can be used for dynamic strain measurement over the short period of time of the strain cycle. The coherent detection is used to beat the Rayleigh signal with a local oscillator at the microwave frequency for phase measurement, and the recovered phase can be converted to strain at the accuracy of submicrometers at a frequency of $500-5 \mathrm{kHz}$ with multiple scans of $\Phi$-OTDR traces at fixed laser frequency [31].

For scanned frequency OTDR trace via tuning laser frequency between two scans of reference and test value, one can also send a chirped pulse which includes multiple frequency components in a single pulse over a pulse length by $\Delta v$ just liked Equation (3.5) at different times of the same frequency spectrum [32]. The Rayleigh pattern change due to temperature or strain will be shifted due to the local refractive index change $\Delta n$. If a $\Delta n$ occurs at a certain location of the fiber $z$, then the local $\Phi$-OTDR pattern will be longitudinally shifted by a $\Delta t$ at that location, correspondent to the $\Delta v$ which compensates for the $\Delta n$. The local $\Delta t$ changes among two different traces can be determined along the fiber by calculating a local cross-correlation of the trace segments obtained for the two consecutive measurements.

\subsection{Distributed Sensor Based on Rayleigh Scattering Enhanced Fiber Devices}

3.4.1. Weak FBGs. The weak Rayleigh scattering signal $(-100 \mathrm{~dB} / \mathrm{mm})$ in the optical fiber has limited the spatial resolution, as the fiber is designed for long haul communications with minimized scattering loss. However, Rayleigh scattering can provide useful information in the sensing system; we can improve Rayleigh scattering by inscribing the grating in the optical fiber to enhance the performance. The strength of the scattering signal in the optical fiber can be controlled by the grating length and the laser power in the grating inscription. The fiber Bragg grating (FBG) has properties of high sensitivity (can be as high as 99\%), compact structure, immunity to electromagnetic interference, and narrow linewidth ranging from sub-nm to $\mathrm{nm}$ in wavelength, which can be multiplexed in the wavelength domain to make a quasidistributed fiber sensor in the wavelength domain using a broadband source [33]. There is a trade-off between grating reflection and total number of the gratings that can be deployed which is limited by the power and bandwidth of the light source. To solve this problem, weak FBGs have been deployed which enable 1000s of identical weak FBGs fabricated in an optical fiber with reflectivity between $-35 \mathrm{~dB}$ and $-40 \mathrm{~dB}$ for each weak grating fabricated during fiber drawing at one peak wavelength [34]. The reflectivity of each FBG affects the multiplexing capacity of a distributed optical fiber sensing system. High reflectivity results in strong spectral-shadowing crosstalk and multiple reflection crosstalks, which will affect the strain or temperature resolution, as well as set a limit for multiplexing capacity. It has been proven that the reflection of $-40 \mathrm{~dB}$ allows suppression of the crosstalk and yet maintains the signal to noise ratio to deploy temperature and strain resolution comparable to the distributed sensor based on SMF [35]. The weak FBG-based sensing system is quasidistributed, because the stress or temperature at optical fibers between the weak FBGs cannot be detected, as the sensing element is weak FBGs. Furthermore, when the length of the grating is decreased, the width of the grating spectrum will be increased. For the limited power over the broad spectrum using optical frequency-domain reflectometry (OFDR) technology [36], the multiplexing grating number will be reduced due to broadband FBGs. In order to solve quasidistributed FBG due to the spatial spacing between weak FBGs, one approach is to use a microcavity array [37]; each pair of adjacent FBGs can form a microcavity to make a true distributed sensor.

Other than static temperature and strain measurement, weak FBGs can also be used for distributed vibration and acoustic sensing. This requires a high speed and high resolution distributed sensing, because the acoustic signal often has a submicrostrain, which means the demodulation system needs to detect nanostrain precision at a $100 \mathrm{kHz}$ sampling rate with $10 \mu$ s speed. One approach is to use a narrow bandwidth filter to translate the wavelength shift measurement to a simple intensity measurement [3]. The system is realized by introducing a reference channel into the demodulation system to compensate for the intensity fluctuation from the light source or optical fiber bending loss with a simple dual channel detection system.

Static and dynamic sensing can be realized by two sets of laser pulses with wavelengths matching the two edges of the FBG reflection spectrum. By using the spectral edges of the gratings, the wavelength shift is converted to the change of intensities at the two wavelengths. With spacing of hundreds of micrometers in FBGs, weak FBGs have broad spectral width of tens of $\mathrm{nm}$, which enables a larger temperature range and strain range. As shown in Figure 6, a dynamic strain sensor is demonstrated by a narrow linewidth laser giving a $3 \mathrm{n} \varepsilon$ accuracy at $50 \mathrm{kHz}$ sampling rate [38].

3.4.2. Random Fiber Gratings. When a telecom fiber is used for distributed fiber optical sensors, the low Rayleigh 


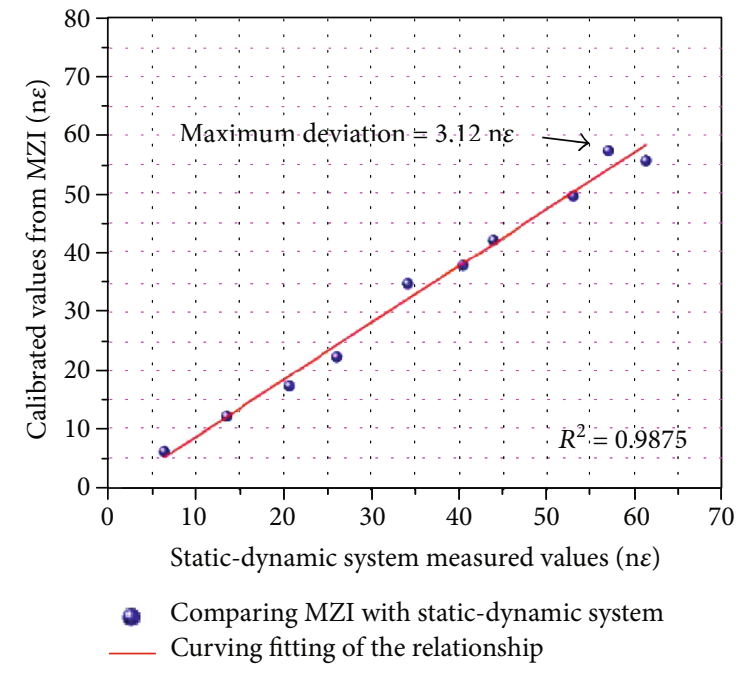

(a)

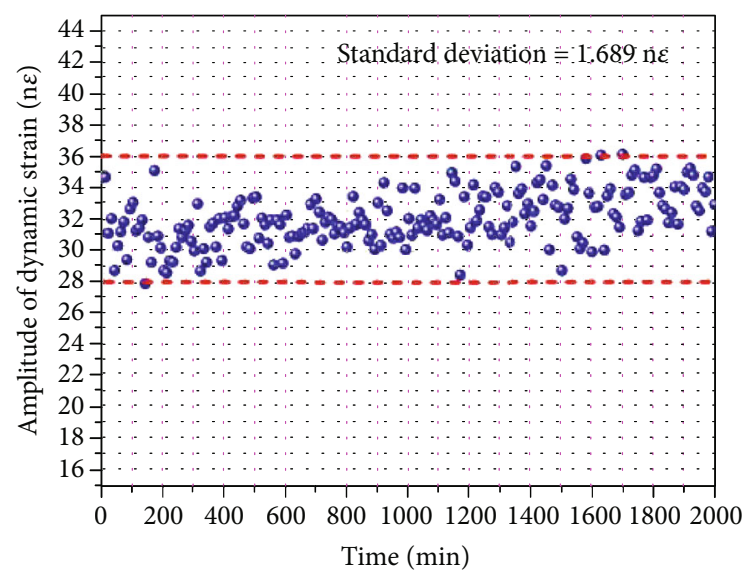

(b)

Figure 6: (a) Comparison between the variation sensing results of our static-dynamic system and a standard MZI. (b) The demodulated results of 200 dynamic strain measurements in the same environment. An interval of 10 minutes is set between each result.

backscattering signal level limits $(-100 \mathrm{~dB} / \mathrm{mm})$ the sensitivity and spatial resolution of distributed temperature or strain measurements by OTDR or OFDR techniques. In recent years, ultraviolet (UV) lasers have been used to increase the backscattering in optical fibers for distributed temperature and strain measurements resulting in higher spatial resolution and high signal to noise ratio (SNR). Using $10.6 \mu \mathrm{m}$ absorption line in SMF-28, the Rayleigh scattering was enhanced by random spaced index modulation using $\mathrm{CO}_{2}$ laser for inscription [39]. With 100 points of $\mathrm{CO}_{2}$-laserirradiation with randomly changed distance along the SMF-28 over a $10 \mathrm{~cm}$ fiber, the random feedback fiber is formed as Rayleigh scattering enhancement. The $10 \mathrm{~cm}$ scattering strength is equivalent to a few $\mathrm{km}$ SMF lengths. The enhancement of Rayleigh scattering is achieved by simple UV exposure of optical fiber. In [40], they used $\mathrm{H}_{2}$ loading on the SMF-28 fiber and specialty fiber with high Ge-dopant to increase UV photosensitivity. Enhancement of Rayleigh scattering with ultralow loss in the SMF28 fiber was demonstrated using specific phase mask [41]; the wavelength range where the backscattering was enhanced was restricted by the specific chirped phase mask used in the experiment. When optical fibers are exposed to high power laser radiation, it would generate broadband loss due to the formation of color centers and/or microvoids that are produced by laser radiation. A novel method to fabricate the random fiber grating by using fs laser is shown in Figure 7 [42].

Using Rayleigh scattering enhanced fiber, the distributed temperature sensor is achieved at an accuracy of $0.00085^{\circ} \mathrm{C}$ over $10 \mathrm{~mm}$ spatial resolution using optical frequency domain reflectometry [42]. The random fiber grating spectrum has spike-like reflective spectra with high contrast that can be attributed to enhanced inhomogeneity. The contrast could be further enhanced when a random fiber grating array is introduced due to the superposition of a few random grating of sub-cm in length. The resultant interference patterns with multiple peaks ensure high accuracy in delay time mea- surement. This outstanding feature resulting in high SNR of Rayleigh traces could be used in the chirped pulse $\Phi$-OTDR system, which is based on the time delay measurement of Rayleigh traces. Unlike a conventional $\Phi$-OTDR sensing system which measures a distributed phase change along the fiber using an ultranarrow linewidth laser, the distributed temperature change-induced time delay is directly measured in real time in chirped pulse $\Phi$-OTDR. This allowed $0.028^{\circ} \mathrm{C}$ accuracy for temperature measurement [4].

3.5. Rayleigh OTDR for Acoustic and Vibration Detection. Section 3.2 provides the theoretical background of measuring temperature and strain based on $\Phi$-OTDR using telecom fiber. The keys to the measurements are as follows: (1) Rayleigh scattering pattern in telecom fiber changes with temperature and strain; (2) Rayleigh pattern also has wavelength dependence; (3) measure the reference pattern at known temperature and strain, sweep the laser wavelength, and recover the Rayleigh pattern at a specific wavelength and then link that wavelength shift to the temperature or strain. Such a procedure is suitable for static measurement. For dynamic measurement, the reference temperature or strain changes with time; it will be difficult to update the associated wavelength change, unless laser wavelength drifting linearly with time is an assumption. Such a statement is often not true, because laser frequency drift can also lead to input polarization state change along the optical fiber and the associated Rayleigh pattern change at a different fiber location, which introduced error [25] in the recovered temperature or strain which is different from the simple calculation Equations (9a) and (9b), especially for vibration and acoustic wave measurement, where the displacement is as small as a few $\mathrm{nm}$ in the spatial domain [43]. According to Equation (9a), for the strain of $5 \mathrm{n} \varepsilon$, the associated laser frequency drift is $0.1 \mathrm{MHz}$ at optical wavelength of $1550 \mathrm{~nm}$. The laser frequency stability over the vibration cycle must be much smaller than $0.1 \mathrm{MHz}$, preferable in the range of 


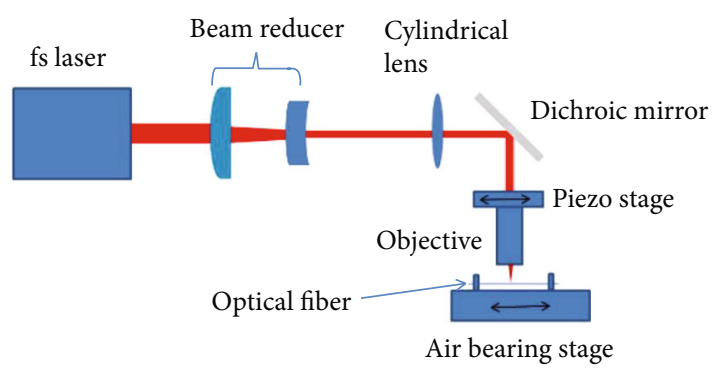

FIgURE 7: The setup of fabricating random fiber gratings.

$\mathrm{kHz}$. As a result, only the narrow linewidth and stabilized laser can be used for dynamic strain of vibration and acoustic wave. However, the vibration frequency information can be obtained with less stringent requirement on the linewidth, because one can use differential Rayleigh scattering traces at two closest times for fast Fourier transfer (FFT) to get the vibration (low frequency) or acoustic wave spectrum [25]. To improve the signal noise ratio for weak Rayleigh scattering, one can use the wavelet denoising method to differentiate small difference between Rayleigh traces at different times, which allows higher frequency $(8 \mathrm{kHz})$ and smaller spatial resolution $(40 \mathrm{~cm})$ detection of vibration or acoustic wave [22] over $1 \mathrm{~km}$ sensing length.

The distributed acoustic sensor was also demonstrated with DFB laser though direct detection [44]; the cyclic pulse coding was introduced to the laser current modulation to enhance performance in order to release the stringent requirement on laser linewidth. However, the laser linewidth and stability must be optimized to simultaneously guarantee intrapulse coherence and interpulse incoherence. The demonstrated frequency for this cost effective laser with coded pulse is $500 \mathrm{~Hz}$ over $5 \mathrm{~m}$ spatial resolution.

Polarization noise in optical fiber can limit distributed acoustic wave detection in phase OTDR; this noise can be mitigated by polarization diversity detection [45]; comparing with coherent detection, polarization diversion detection can bring $10 \mathrm{~dB}$ SNR improvement for the same power level and spatial resolution. This technique mitigates the polarization mismatch between the sensing arm and reference arm along the sensing fiber, which is more suitable for the multievent detection in distributed acoustic sensing.

3.6. Chirped Pulse-Based $\Phi-O T D R$. Most recently, chirped pulse phase OTDR is introduced for acoustic sensing; instead of using transform limited pulse, it used a train of linearly chirped optical pulses [32]. In this technique, the local strain variation due to acoustic disturbance accumulated between two temporal instants and is directly measured in each spatially resolved point. Hence, local strain transduces a local fiber perturbation into a local disturbance of the optical trace via refractive index change; such information can be recovered in time domain directly via time delay measurement for strain change. The chirped pulse with spectral range $\Delta$ $v_{p}$ and pulse width $\tau_{p}$. The frequency-to-time mapping gives a perturbation-induced spectral shift $\Delta v$ over the chirped wavelength range which can be translated to a local temporal delay $\Delta t[46]$.

$$
\Delta t=-\frac{\tau_{p}}{\delta v_{p}} \Delta v
$$

Chirped pulse can also be recovered by phase change, which involves demodulation of the phase information though $\phi_{i}=\left(4 \pi\left(n L_{i}\right) / c\right) v=\left(4 \pi\left(n^{\prime} L_{i}^{\prime}\right) / c\right) v^{\prime}$, where $n^{\prime} L^{\prime}$ represents local optical path length change from $n L$ at position $L^{\prime} ; v^{\prime}$ is the changed optical frequency from $v$. In chirped pulse $\Phi$-OTDR, or general $\Phi$-OTDR, it allows faster sensitive measurements by phase recovered information, which is a relative measurement; when the temperature or strain of the fiber is changed in one position, it will inevitably accumulate an error for long integration times.

In the conventional chirped pulse $\Phi$-OTDR system, the maximum measurable strain or temperature variation is limited by the large time delay estimation (TDE) error due to the distortions of reflected traces from the sensing location. In [47], a high performance distributed sensing system based on random fiber grating array (RFGA) and multifrequency database demodulation (MFDD) method for strain-induced delay time measurement is demonstrated. The MFDD method is proposed and operated as follows: Firstly, a database including several traces without perturbation is established. These traces are collected when chirped pulses with different initial optical frequency are used to interrogate the whole RFGA. Secondly, coded traces are recorded using chirped pulses with known fixed initial optical frequencies when dynamic strain is applied in a period of time. Thirdly, in the demodulation process, the cross-correlation calculation between each encoded trace and each reference trace in the database is employed. Finally, the cross-correlation calculation result of each coded trace which possesses the highest coefficient will be selected for relative strain change determination. The overall strain variation is reconstructed based on the initial frequency difference and time delay without integral operation. It enables a wide measurement range for both static and dynamic strain sensing, since the TDE accuracy is maintained in high value in large strain variation by introducing initial frequency difference compensation. Because of the requirement of high temporal stability and large optical frequency scanning range for data base establishment, a random fiber grating array is used as the sensor part to provide stable reflection with wide spectrum range. Finally, a dynamic strain with peak-to-peak value of $12.5 \mu \varepsilon$ at vibration frequencies of $10 \mathrm{~Hz}$ and $50 \mathrm{~Hz}$ is accurately reconstructed as shown in Figure 8 when the pulse repetition rate is $1 \mathrm{kHz}$, which was not detected using a conventional chirped pulse $\Phi$-OTDR. The maximum measurable strain variation of about $12.5 \mu \varepsilon$ represents a three-times improvement. This number is limited by prerecorded frequency scanning range of RFGA response in the database.

3.7. $\Phi-O T D R$ for Laser Phase Noise Characterization. Narrow linewidth laser is essential for high precision optical metrology and coherent communication. The characterization of 


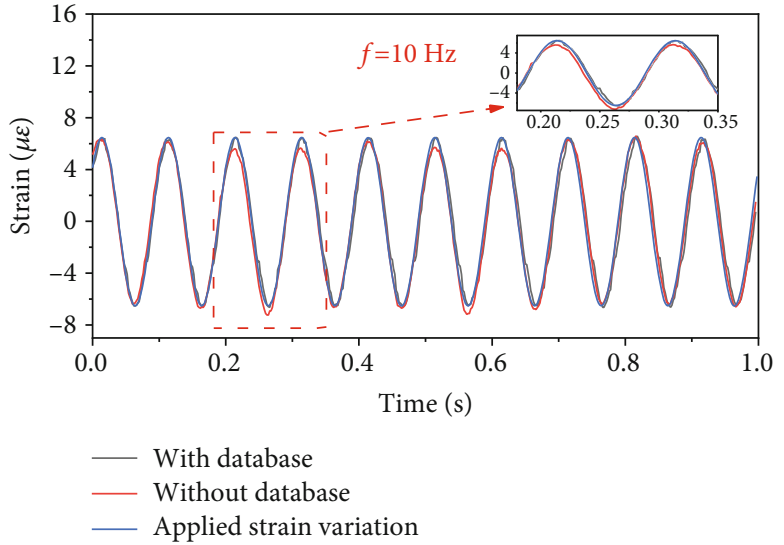

(a)

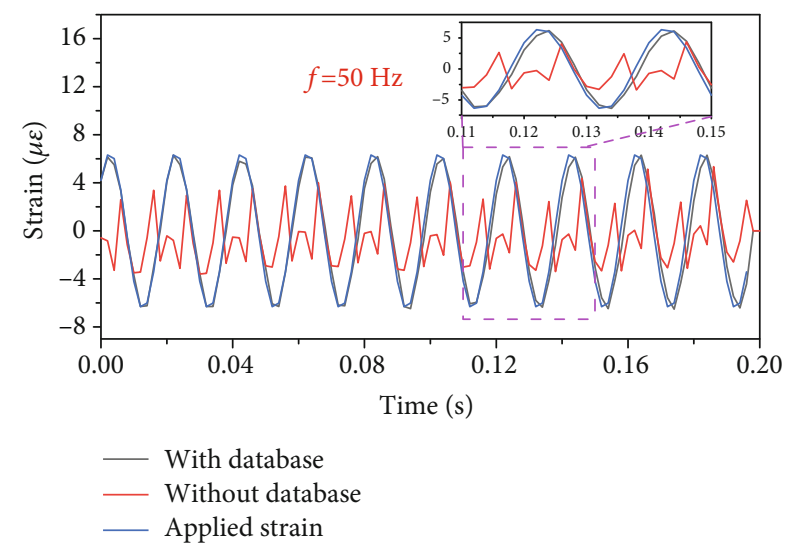

(b)

Figure 8: Demodulated dynamic strains of two different methods (with and without database): (a) $10 \mathrm{~Hz}$ sinusoidal variation; (b) $50 \mathrm{~Hz}$ sinusoidal variation.

the low phase noise, especially for linewidth $\Delta v_{L}$ of sub-kHz $\Delta v_{L}<1 \mathrm{kHz}$ is often carried by heterodyne detection of beating frequency of the test laser and a reference laser with linewidth much smaller than test laser, for instance, $\Delta v_{\mathrm{L}}<50 \mathrm{kHz}$. Such a super narrow linewidth reference laser is not often available in normal labs. Alternatively, one can use delayed self-heterodyne interferometer (DSHI) method to decorrelate the laser beam over the delay length longer than the laser coherent length and recombine them [48]. For the laser linewidth of sub-kHz, say $500 \mathrm{~Hz}$, the coherent length will be $400 \mathrm{~km}$, which gives $80 \mathrm{~dB}$ loss at wavelength of $1550 \mathrm{nmb}$ with minimum loss. The introduction of fiber amplifier via loss-compensated recirculating technique will give an extended long delay [49], and at the same time, it will add the time-dependent noise to measured linewidth; for the small phase noise laser, the added noise could be comparable to the tested laser itself. Hence, the DSHI method is inappropriate for sub-kHz linewidth laser.

Fortunately, coherent detection of $\Phi$-OTDR itself can be used to characterize the narrow linewidth laser [50]. The pump laser is the tested laser for $\Phi$-OTDR; the phase variance with time can be recovered as a function of the position (time) via coherent detection. The phase jitter of the phase variance can be improved by the multiple pulses to reduce uncertainty for linewidth measurement. The delay time $\tau$ and the measured phase jitter variance $\sigma_{\tau}^{2}$ of the test laser linewidth $\Delta v_{c}$ has linear slope of $\sigma_{\tau}^{2}=2 \pi \Delta v_{c} \tau$. Note the time interval $\Delta t$ (in ms range) between two consecutive traces for beating note traces should be longer than the coherent time of the laser in order to mitigate any correlation between them for independent statistical analysis of the phase jitter variance. The paper demonstrated the following laser linewidths: $554.0( \pm 10.0) \mathrm{Hz}$ and $998.1( \pm 23.0) \mathrm{Hz}$ using $1 \mathrm{~km}$ Rayleigh scattering fiber, which demonstrated an intrinsic linewidth measurement via statistical analysis of laser phase noise, as shown in Figure 9.

3.8. Challenge in Rayleigh Scattering-Based OTDR Sensing. The evaluation of the Rayleigh scattering-based $\Phi$-OTDR

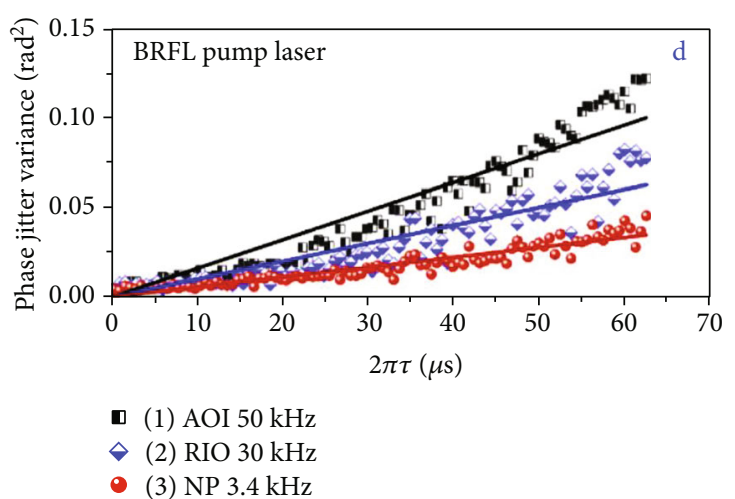

FIGURE 9: Investigation of BRFL pump laser impact on phase noise statistics and intrinsic linewidth. The BRFL was pumped by three different pump lasers: (1) AOI laser with $50 \mathrm{kHz}$ (DFB, AOI Inc.), (2) RIO laser with $30 \mathrm{kHz}$ (RIO009x, RIO Inc.), and (3) NP laser with $3.4 \mathrm{kHz}$ (Rock Module, NP Photonics Inc.). Accordingly, the intrinsic linewidths of the BRFLs with three pump lasers were mathematically obtained as $554.0( \pm 10.0) \mathrm{Hz}, 998.1( \pm 23.0) \mathrm{Hz}$, and $1600.0( \pm 30.2) \mathrm{Hz}$, respectively.

as distributed fiber sensors for structural health monitoring (SHM) includes the following factors.

3.8.1. Sensing Length and Spatial Resolution. Sensing range is directly linked to the area of the sensing coverage in SHM, while spatial resolution determines the accuracy of the locating abnormal frequency spot, for instance, deformation of the oil pipeline has a leaky point or internal crack location. There is a trade-off between the sensing length and the spatial resolution, simply because the increased sensing length leads to the high noise accumulated over the sensing length and fiber attenuation, although the fiber amplifier, such as EDFA, can bring the overall signal level; however, EDFA itself also introduces noise and it does not improve the signal to noise ratio.

Under the smallest spatial resolution, the disturbance of the dynamic strain could be combined with the coherent 
Rayleigh signal from the entire fiber length as a noise floor. In this situation, one can use the Kerr nonlinear effect to magnify the changed signal (sensing element) and remove the large DC background signal [51]. The mechanism is a parametric amplification via the signal and the pump, creating two sidebands at higher orders for amplification.

The noise floor contribution from coherent Rayleigh scattering along the entire optical fiber is due to the finite extinction ratio of the EOM $(<25 \mathrm{~dB})$ with high extinction ratio $\mathrm{EOM}(<40 \mathrm{~dB})$; the noise floor can be removed by high extinction ratio optical pulse generation $(>60 \mathrm{~dB})$ by adding to the high extinction ratio EOM together for optical pulse generation [52]. The highest ER pulses in the report are over $100 \mathrm{~dB}$ [53]. The low noise floor from high ER pulse enables a $26.5 \mathrm{~km}$ sensing length to detect $2 \mathrm{~m}$ vibration signals without optical amplification. The shortest spatial resolution by phase-sensitive OTDR is $50 \mathrm{~cm}$ [22], which was achieved by continuous wavelet transform for nonstationary vibration measurement.

Using Raman optical amplifier for both of pump power amplification and backscattering Rayleigh signal amplification, the longer sensing length with Rayleigh OTDR has been demonstrated with $50 \mathrm{~km}$ sensing length, where a $60 \mathrm{~m}$ long PZT stretched fiber is used to provide a sinusoidal strain at $2 \mathrm{~Hz}$ for the strain of the $80 \mathrm{n} \varepsilon$ peak to peak value [46]. $100 \mathrm{~km}$ sensing range is possible with 20 averages of the optical trace and bidirectional Raman amplification (copropagating Raman pump at $300 \mathrm{~mW}$, counterpropagating Raman pump at $380 \mathrm{~mW}$ ). Although increasing the pump power for the Raman signal in the bidirectional direction may allow longer sensing length. However, the signal to noise ratio is not infinitive for a given pump power, and hence, a longer fiber length can be achieved at the cost of the spatial resolution.

3.8.2. Acoustic or Vibration Frequency Range. The acoustic frequency spectra can be linked to the condition of the internal crack or deformation in the pipeline, bridge, power generator, airplane, rail, etc.; the size of the crack or deformation can be related to the measured acoustic or vibration frequency; and the smallest dimension corresponds to the highest frequency. The structural health monitoring of civil structures and damage detection in mechanical processes require frequency detection from a few $\mathrm{Hz}$ to hundreds of $\mathrm{kHz}$, even $\mathrm{MHz}$ [43].

The maximum measurable acoustic frequency $f_{\max }$ in the OTDR sensor system is limited by the repetition rate of the pulse signal. This limit is set to avoid overlapping of the optical traces by consecutive optical pulses, i.e., at any given time, there will be one optical pulse traveling in an optical fiber at the speed of light as $c / n_{g}$ from one end to the other end as a probe signal and return to the starting point as a backscattering Rayleigh signal. This gives twice of the fiber length $L$ for $f_{\max }$ :

$$
f_{\text {max }}=\frac{1}{2} \times \frac{c}{2 n_{g} L} .
$$

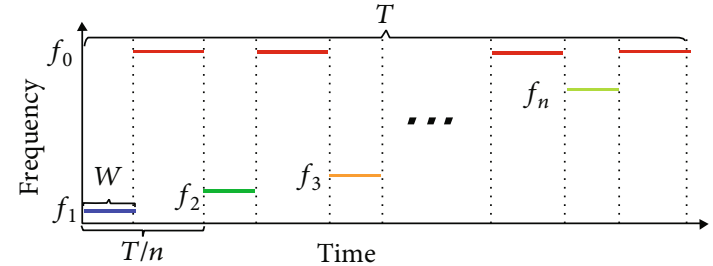

FIGURE 10: Frequency coding of the test pulse sequence.

The factor $1 / 2$ is introduced by the Nyquist theorem. The high frequency detection may be available by frequency-multiplexed OTDR, which allows detection of the entire distribution of a high-frequency vibration [54]. They used dense frequency division multiplexed OTDR (FDM-OTDR) with one modulator to enhance the sensitivity of coherent OTDR. The technique is to modify the frequency encoding for high-speed phase monitoring with control software parameters as shown in Figure 10. It includes multiple frequency components $f_{i}(i=1, \cdots, n)$ with a duration $W$ and frequency spacing $\Delta f$ which is separated by $T / n$ in the time domain, which is also the sampling rate for vibration detection in FUT between the frequency components in the pulse sequence. $T$ is the total duration of the pulse sequence, which is designed to be larger than the round trip time in FUT for $1 / T$, so that the same frequency components would not occur in FUT. The integral number $n$ is a multiplexing number which is limited by the detection bandwidth for single round trip time system design. The frequency demultiplexing and signal processing can be realized by software processing via short-time Fourier transformation (STFT) to separate multiple frequency components into multiple OTDR traces. The sampled data for STFT corresponds to the duration $W$, which means distance in OTDR traces.

3.8.3. Smallest Measurable Temperature and Strain. The highest sensitivity for stress measurement is associated with the depth of the crack or deformation. For 3D mapping of the deformation, the information of the displacement of dynamic strain and frequency spectrum could work together to provide conditional monitoring for a structure using finite element analysis.

The smallest measurable displacement is also called dynamic strain, which is limited by minimum distributed phase information. Such a measurement is limited by noises from the laser noises including laser frequency drift and relative intensity noise (RIN), which can be minimized by using a narrow linewidth and stabilized laser source. However, it will increase the overall budget for a phase OTDR-based distributed sensor system. Secondly, the smallest measurable value is also limited by the fiber polarization noises, which can be minimized by using a polarization marinating fiber (PMF) configuration [27], which can be expansive if large structural monitoring is needed, i.e., a few km PMF. Alternatively, one can use the telecom fiber with polarization diversity detection scheme [17].

To correct overall noise from laser and telecom fibers, one can use homodyne phase demodulation scheme, which 
is based on selective phase demodulation of one of the double pulse probe and a direct detection for dynamic phase demodulation [55]. The continuous wavelet transformation is also introduced to the recovered time-dependent frequency spectrum [22].

The minimum detectable dynamic strain with Rayleigh OTDR can be as small as pe [56] for the noise floor of $100 \mathrm{p} \varepsilon / \sqrt{ } \mathrm{Hz}$ with repletion rate of $\mathrm{kHz}$. Such a small strain measurement is enabled by wavelength scanning coherent OTDR. The minimum detectable strain for single wavelength Rayleigh OTDR is $80 \mathrm{n} \varepsilon$ [31], which is 1000 times larger than the wavelength scanning OTDR result. The reason for such a difference is that the phase recovery signal for a single wavelength source is strongly affected for the laser wavelength drift and phase noise, while in the large wavelength range, phase demodulation is an enveloped change, which has little dependence on individual wavelength stability. The crosscorrelation over a wavelength range takes large average effect; depending on the wavelength range, the equivalent single wavelength traces have an average of $10^{4}-10^{6}$. Hence, it is not surprising to see much smaller strain accuracy in chirped pulse OTDR comparing with single wavelength pulse OTDR. The advantage of the single wavelength OTDR for strain or temperature measurement is the significantly large range. While in chirped pulse OTDR, when the strain or temperature is changed relative to the reference values, the correlation calculation between two large strains or temperatures can lead to two differently shaped spectrums, which makes them decorrelated. This will be the range for the maximum range.

\section{Optical Frequency Domain Reflectometry (OFDR)}

The basic of the OFDR sensor can be found in two review papers $[5,43]$. In this section, we will add some new development in recent years. OFDR utilizes a tunable laser and an interferometer to superpose a swept-laser signal with a time-delayed version of the same signal leading to a beat. The demodulation of the OFDR sensor includes two functions: (1) wavelength detection to compensate nonlinearity in laser wavelength tuning and (2) Mach-Zehnder interferometer for heterodyne detection of the laser tuning frequency and delayed Rayleigh signal at different wavelengths; the Rayleigh signal as a function of the beat signal changes with temperature and strain due to optical path length change.

To record this beat signal change as a function of time, the laser coherence length should be comparable to the sensing fiber length [57]. Beyond of the coherence length, there are many random noises in various positions along the sensing length, which sets the sensing length limitation for OFDR sensors. Such a problem can be resolved by introducing phase compensation by software processing, which allows the sensing range to enlarge from $50 \mathrm{~m}$ to $350 \mathrm{~m}$ for static strain or temperature measurement [58]; beyond software processing to improve the OFDR sensing range, hardware signal processing has been proposed, such as self-induced phase modulation by the nonlinear Kerr effect over long range reflectometers [59]. This enables overcoming the laser coherence length to detect a PMF circulator with a connector end at $201 \mathrm{~km}$ sensing length for the spatial resolution of $13 \mathrm{~cm}$ without any fiber amplifier being introduced. The confirmed laser coherence length is $10 \mathrm{~km}$ [60]. Clearly, the detection is far beyond that of the laser coherence length.

Another approach to increase the sensing length limit due to interferometry fading from the finite laser coherence length is to use time-gated digital OFDR; it used a narrow linewidth laser, followed by an AOM to create a long pulse; within the long pulse, there is a SAW wave to provide frequency sweeping [61]. The detection is coherence detection with a 90-degree optical hybrid to extract phase information to recover vibration signal at $3.5 \mathrm{~m}$ spatial resolution over $40 \mathrm{~km}$ fiber length. With further improvement in phase detection and fading noise compensation of this proposed technique, $110 \mathrm{~km}$ sensing length with $1.6 \mathrm{~m}$ spatial resolution was demonstrated [62].

The first dynamic strain measurement using OFDR was proposed and demonstrated by time-resolved OFDR [63], which is based on a different time segment of wavelength range to recover beat frequency as a function of time and location. Because of the smaller wavelength range, the spatial resolution was compromised of a few $\mathrm{cm}$ with frequency range of less than $50 \mathrm{~Hz}$. Using PMF, one can use relative change of fast and slow axis to remove the need of reference trace for real-time dynamic strain measurement using OFDR [64], which allows $200 \mathrm{n} \varepsilon$ being detected. The maximum detectable dynamic strain is limited by the positiondependent Rayleigh scattering pattern correlation in OFDR traces, i.e., backscattered traces depend on the spatial distribution of the inhomogeneity in the sensing fiber [65]. To overcome this limit, the position calibration is for spectrum registration was proposed to reduce the deterioration induced by the position segment mismatch in the spatial domain; this allowed the $7000 \mu \varepsilon$ range [66].

The OFDR sensor can only measure either temperature or strain for the Rayleigh pattern shift. To separate temperature and strain, one can use PMF to measure birefringenceinduced wavelength shift in fast and slow axes, which allows simultaneous temperature and strain measurements with $0.8^{\circ} \mathrm{C}$ temperature resolution and $7 \mu \varepsilon$ strain accuracy for spatial resolution of $6 \mathrm{~mm}$ [67]. Alternatively, one can also use two types of the fibers, such as a standard single mode fiber (SMF) and a reduced-cladding SMF by Rayleigh backscattering spectra shifts in OFDR; it allows $18 \mathrm{~cm}$ spatial resolution for temperature resolution of $0.3^{\circ} \mathrm{C}$ and strain resolution of $8 \mu \varepsilon$ [68].

\section{Challenges and Limitations in Distributed Fiber Optic Sensors}

Scattering-based OTDR sensor is capable for long sensing length, because of low scattering loss; the pulsed signal can be transmitted over tens of kilometers or up to $200 \mathrm{~km}$ in length. However, the returned backscattering signal can be very weak depending on the spatial resolution of the Rayleigh, Brillouin, or Raman scattering; the scattering signal level can be in the range of $-40 \mathrm{~dB}$ to $-80 \mathrm{~dB}$ plus fiber attenuation which is a function of total sensing length. 


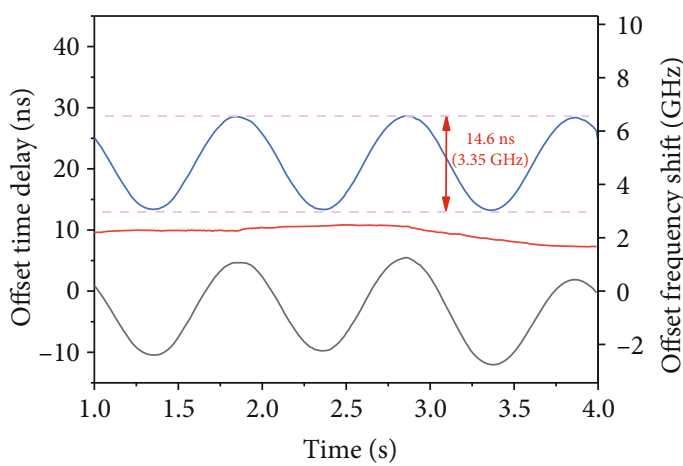

After compensation
- Compensation curve
- Before compensation

(a)

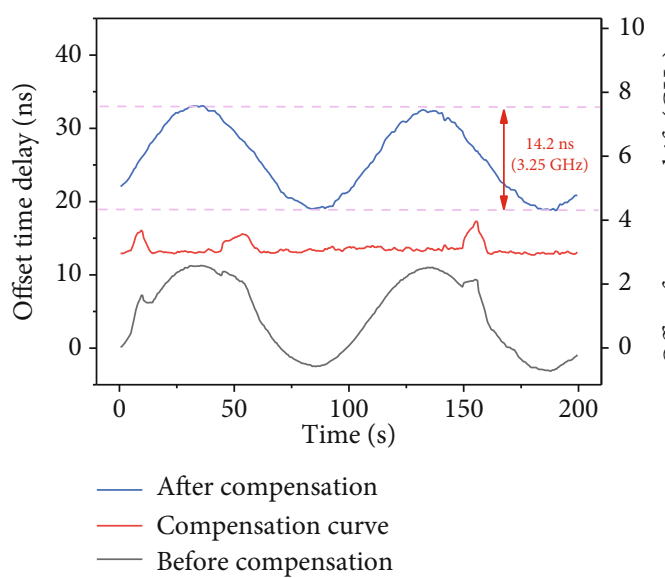

(c)

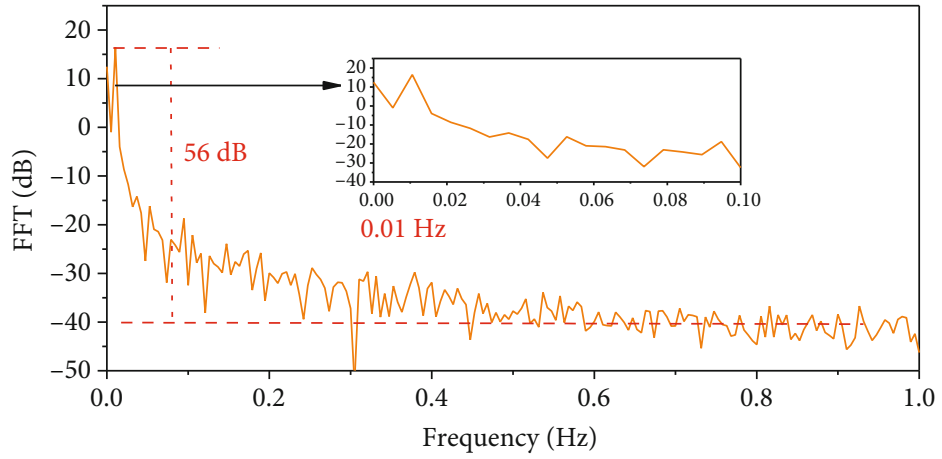

— FFT of reconstructed signal

(b)

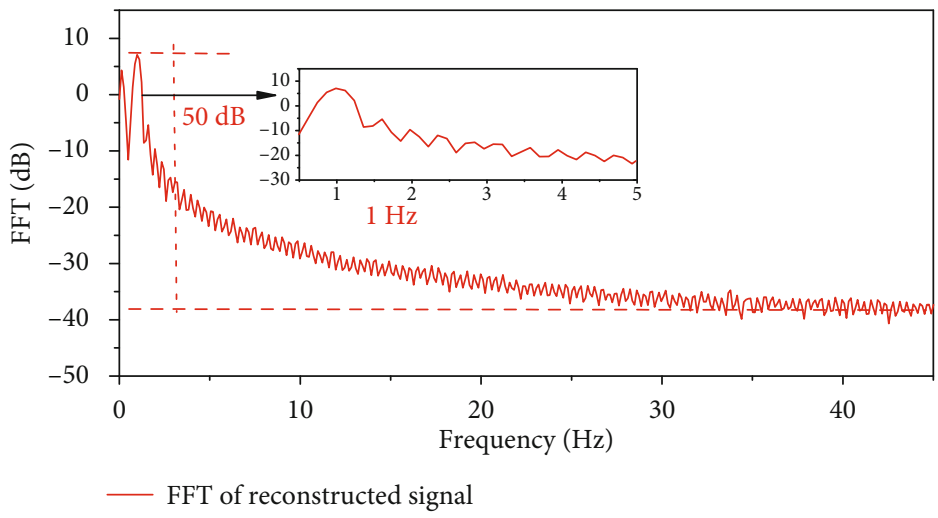

(d)

FIGURe 11: Dynamic strain measurement with ultralow vibration frequency of (a) $1 \mathrm{~Hz}$ and (c) $0.01 \mathrm{~Hz}$ and its FFT spectra (b, d).

The sensing range limitation due to a weak signal at the end of the fiber in principle can be boosted by optical amplifiers, for instance, EDFA or Raman amplifiers; however, those amplifiers also amplify noise at the same time in addition to adding 4-6 dB noise, which reduces the signal to noise ratio. In this situation, one can use the Kerr effect to magnify the small signal before the detection system [51]; this method can bring the weak signal to the detectable level, which can be helpful in improving the spatial resolution, as it allows a smaller pulse with shorter spatial resolution for distributed sensors, so that the effective signal to noise ratio will be improved with the same DC offset signal. In [69], to achieve long-haul measurement without distributed amplification, the distortion of the amplified pulse is rectified by using iterative predistortion method to boost the pulse energy and suppress the self-phase modulation; besides, a three-carrier pulse is employed to mitigate the influence of the interference fading and the stimulated Brillouin backscattering.

Another limitation is the maximum detectable frequency range which is limited by the fiber length. A higher frequency response of up to $3 \mathrm{MHz}$ is achieved in [70]. The proposed system-combined benefits of MZI and $\Phi$-OTDR allow it for high-frequency responses with satisfied spatial resolution.
In addition, by impressing vibration signals on a set of backscattering signals that are carried by different wavelengths within one measurement interval, the detectable frequency response range also could be enhanced [71]. Another method to break the limitation of the roundtrip time of the light pulse is that the pulse interval is randomly modulated so that an equivalent sub-Nyquist additive random sampling (sNARS) is realized for every sensing point of the long interrogation fiber [72].

In the $\Phi$-OTDR system, significant laser frequency drifting (LFD) has limited the detection in the low frequency range $(0.01 \mathrm{~Hz}-20 \mathrm{~Hz})$, where the $\mathrm{LFD}$ will introduce a time-dependent noise destroying the dynamic strain reconstruction. In [73], a simple and effective method that utilizes the referenced random fiber grating to monitor the variation of laser frequency is proposed. As shown in Figure 11(c), during the total measurement time of $200 \mathrm{~s}$, the laser frequency drifting-induced time delays are accurately monitored by the random fiber grating array. And then the reconstructed dynamic strain profile without the laser frequency drifting noise is obtained.

The structural health monitoring in field request multiparameter sensing in a distributed way, for instance, 
temperature, strain, vibration, and acoustic wave should be measured simultaneously. The key challenge is to find a system that will enable the physical parameters to be determined at any point along the sensing fiber with high sensitivity and spatial resolution, yet within acceptable frequency range for dynamic vibration, strain, and temperature detection. There are many applications, especially in geophysical and mining engineering, where simultaneous measurement of vibration and temperature is essential [74].

One example is the time-lapse measurements of temperature, vibration, and strain in and around the wellbore, which provides critical reservoir information, and they can be used for production and recovery optimization. To get that information, the end users may have to use three sensor systems based on Rayleigh scattering and Brillouin scattering, as well as pressure sensor. The individual distributed systems require additional fiber-optic cables, equipment, installations, and acquisition of three different large data sets. This adds unnecessary tasks to the overall reservoir management work flows, increases cost, and ultimately limits applications of the technology in the future well and reservoir surveillance. In today's technology, using a weak FBG array-based distributed sensor, one can get a single sensor system to realize dynamic and static measurements [38]; the multiplexing technique will allow thousands of the distributed sensors to be deployed inside of the fiber [37].

The new development of ultraweak FBGs and random grating array sensors has enabled the tens of meters to a few kilometers of the sensors to be detected for simultaneous multiparameter sensing with spatial resolution of $\mathrm{mm}$ to $\mathrm{cm}$. The strain accuracy can have sub- $\mu \mathrm{m}$ level, which is certainly in the level of the acoustic wave detection range. They can be used for mine safety and operations, leak detection in the pipeline and abandoned gas wells, induced microseismic monitoring, and bridges.

\section{Conclusion}

Distributed fiber sensor research has achieved a lot of exciting developments over the last 10 years, especially in the field application for health monitoring of civil and geoengineering structures. As they offer a number of distinguishing advantages, great advancements have been achieved in recent years in terms of long sensing length $(200 \mathrm{~km})$, high spatial resolution $(\mathrm{mm}$ to $1 \mathrm{~cm})$ and high strain, temperature, and acoustic and vibration resolution (nanostrain resolution, less than $0.01^{\circ} \mathrm{C}$ temperature resolution, sub- $\mathrm{Hz}$ to $\mathrm{MHz}$ acoustic wave detection). This has proven them to be one of the most promising candidates for fiber-optic smart structures.

This review presents a comprehensive and systematic overview of the history of distributed sensor technology regarding many aspects including sensing principles, properties, and their performance, system limitations, and applications. It is anticipated that many more distributed sensor systems will be commercialized and widely applied in practice in the near future due to the maturity of many new technologies and the availability of cost effective instrumentation techniques.

\section{Conflicts of Interest}

The authors declare that there is no conflict of interest regarding the publication of this article.

\section{Authors' Contributions}

X.B. wrote the manuscript with support from Y.W. Both X.B. and Y.W. contributed to the final version of the manuscript.

\section{Acknowledgments}

This research was funded by the Natural Sciences and Engineering Research Council of Canada, grant number RGPIN-2020-06302, and Canada Research Chairs, grant number 950231352.

\section{References}

[1] M. Mitra and S. Gopalakrishnan, "Guided wave based structural health monitoring: a review," Smart Materials and Structures, vol. 25, article 053001, 2016.

[2] M. K. Barnoski and S. M. Jensen, "Fiber waveguides: a novel technique for investigating attenuation characteristics," Applied Optics, vol. 15, no. 9, pp. 2112-2115, 1976.

[3] P. Han, Z. Li, L. Chen, and X. Bao, "A high-speed distributed ultra-weak FBG sensing system with high resolution," IEEE Photonics Technology Letters, vol. 9, no. 15, pp. 1249-1252, 2017.

[4] Y. Wang, P. Lu, S. Mihailov, L. Chen, and X. Bao, "Distributed time delay sensing in a random fiber grating array based on chirped pulse $\varphi$-OTDR," Optics Letters, vol. 45, no. 13, pp. 3423-3426, 2020.

[5] X. Bao and L. Chen, "Recent progress in distributed fiber optic sensors," Sensors, vol. 12, no. 7, pp. 8601-8639, 2012.

[6] S. A. Kingsley and D. E. N. Davies, "OFDR diagnostics for fibre and integrated-optic systems," Electronics Letters, vol. 21, no. 10 , pp. 434-435, 1985.

[7] B. E. A. Saleh and M. C. Teich, Fundamentals of Photonics, Wiley-VCH, 2019.

[8] N. Nakazawa, "Rayleigh backscattering theory for single-mode optical fibers," Journal of the Optical Society of America, vol. 73, no. 9, pp. 1175-1180, 1983.

[9] G. Agrawal, Fiber-Optic Communication Systems, Wiley, 2002.

[10] E. Brinkmeyer, "Analysis of the backscattering method for single-mode optical fibers," Journal of the Optical Society of America, vol. 70, no. 8, pp. 1010-1012, 1980.

[11] G. Oscroft, "Intrinsic fiber optic sensors," in Proceedings of International Conference on Fibre Optics and Opto-Electronics, pp. 207-213, London, UK, 1987.

[12] E. Marcuse, "Microbending losses of single-mode, step-index and multimode, parabolic-index fibers," Bell System Technical Journal, vol. 55, no. 7, pp. 937-955, 1976.

[13] A. Hartog, "A distributed temperature sensor based on liquidcore optical fibers," Journal of lightwave Technology, vol. 1, no. 3, pp. 498-509, 1983.

[14] M. C. Farries, M. E. Fermann, R. I. Laming, S. B. Poole, and D. N. Payne, "Distributed temperature sensor using $\mathrm{Nd} 3+$ -doped optical fibre," Electronics Letters, vol. 22, no. 8, pp. 418-419, 1986. 
[15] M. C. Farries, M. E. Fermann, S. B. Poole, and J. E. Townsend, "Distributed temperature sensor using holmium 3+ doped fiber," in Proceedings of OFC, Reno, NV, USA, 1987.

[16] W. Seo, Fiber Optic Intrusion Sensor Investigation, 1994.

[17] M. Ren, Distributed optical fiber vibration sensor based on phase-sensitive optical time domain reflectometry, MSc thesis, Université d'Ottawa/University of Ottawa, 2016.

[18] L. Shi, T. Zhu, Q. He, and S. Huang, "Effect of laser linewidth on phase-OTDR based distributed vibration sensing regime," in OFS2014 23rd International Conference on Optical Fiber Sensors, Santander, Spain, 2014.

[19] X. Zhong, C. Zhang, L. Li et al., "Influences of laser source on phase-sensitivity optical time-domain reflectometer-based distributed intrusion sensor," Applied Optics, vol. 53, no. 21, pp. 4645-4650, 2014.

[20] F. Zhu, X. Zhang, L. Xia, Z. Guo, and Y. Zhang, "Active compensation method for light source frequency drifting in $\Phi$ -OTDR sensing system," IEEE Photonics Technology Letters, vol. 27, no. 24, pp. 2523-2526, 2015.

[21] X. Hui, S. Zheng, J. Zhou et al., "Electro-optic modulator feedback control in phase-sensitive optical time-domain reflectometer distributed sensor," Applied Optics, vol. 52, no. 35, pp. 8581-8585, 2013.

[22] Z. Qin, L. Chen, and X. Bao, "Wavelet denoising method for improving detection performance of distributed vibration sensor," IEEE Photonics Technology Letters, vol. 24, pp. 542-544, 2012.

[23] J. C. Juarez and H. F. Taylor, "Field test of a distributed fiberoptic intrusion sensor system for long perimeters," Applied Optics, vol. 46, no. 11, pp. 1968-1971, 2007.

[24] J. C. Juarez, E. W. Maier, K. N. Choi, and H. F. Taylor, "Distributed fiber-optic intrusion sensor system," Journal of Lightwave Technology, vol. 23, no. 6, pp. 2081-2087, 2005.

[25] Y. Lu, T. Zhu, L. Chen, and X. Bao, "Distributed vibration sensor based on coherent detection of phase-OTDR," Journal of lightwave Technology, vol. 28, p. 3243, 2010.

[26] D. W. Stowe, D. R. Moore, and R. G. Priest, "Polarization fading in fiber interferometric sensors," IEEE Transactions on Microwave Theory and Techniques, vol. 30, pp. 1632-1635, 1982.

[27] Z. Qin, Z. Tao, L. Chen, and X. Bao, "High sensitivity distributed vibration sensor based on polarization-maintaining configurations of phase-OTDR," IEEE Photonics Technology Letters, vol. 23, pp. 1091-1093, 2011.

[28] A. D. Kersey, M. J. Marrone, and A. Dandridge, "Polarization diversity detection for fiber interferometers using active feedback control of output polarization-mode selection," Optics Letters, vol. 15, no. 22, pp. 1315-1317, 1990.

[29] Y. Koyamada, M. Imahama, K. Kubota, and K. Hogari, "Fiberoptic distributed strain and temperature sensing with very high measurand resolution over long range using coherent OTDR," Journal of Lightwave Technology, vol. 27, no. 9, pp. 1142-1146, 2009.

[30] J. Posey, G. A. Johnson, and S. T. Vohra, "Strain sensing based on coherent Rayleigh scattering in an optical fibre," Electronics Letters, vol. 36, no. 20, pp. 1688-1689, 2000.

[31] A. Masoudi, M. Belaland, and T. P. Newson, "A distributed optical fibre dynamic strain sensor based on phase-OTDR," Measurement Science and Technology, vol. 24, no. 8, article 085204, 2013.
[32] J. Pastor-Graells, H. F. Martins, A. Garcia-Ruiz, S. MartinLopez, and M. Gonzalez-Herraez, "Single-shot distributed temperature and strain tracking using direct detection phasesensitive OTDR with chirped pulses," Optics Express, vol. 24, no. 12, pp. 13121-13133, 2016.

[33] D. Kinet, P. Mégret, K. W. Goossen, Q. Liang, D. Heider, and C. Caucheteur, "Fiber Bragg grating sensors toward structural health monitoring in composite materials: challenges and solutions," Sensors, vol. 14, no. 4, pp. 7394-7419, 2014.

[34] H. Guo, J. Tang, X. Li, Y. Zheng, H. Yu, and H. Yu, "On-line writing identical and weak fiber Bragg grating arrays," Chinese Optics Letters, vol. 11, article 030602, 2013.

[35] Y. Wang, J. Gong, D. Y. Wang, B. Dong, W. Bi, and A. Wang, "A quasi-distributed sensing network with time-divisionmultiplexed fiber Bragg gratings," IEEE Photonics Technology Letters, vol. 23, p. 70, 2010.

[36] H. Igawa, K. Ohta, T. Kasai, I. Yamaguchi, H. Murayama, and K. Kageyama, "Distributed measurements with a long gauge FBG sensor using optical frequency domain reflectometry," Journal of Solid Mechanics and Materials Engineering, vol. 73, no. 724, pp. 1912-1920, 2008.

[37] C. Wang, Z. Li, X. Gui et al., "Micro-cavity array with high accuracy for fully distributed optical fiber sensing," Journal of Lightwave Technology, vol. 37, no. 3, pp. 927-932, 2019.

[38] Z. Li, Y. Tong, X. Fu et al., "Simultaneous distributed static and dynamic sensing based on ultra-short fiber Bragg gratings," Optics Express, vol. 26, no. 13, pp. 17437-17446, 2018.

[39] Y. Li, P. Lu, X. Bao, and Z. Ou, "Random spaced index modulation for a narrow linewidth tunable fiber laser with low intensity noise," Optics Letters, vol. 39, no. 8, pp. 2294-2297, 2014.

[40] S. Loranger, M. Gagné, V. Lambin-Iezzi, and R. Kashyap, "Rayleigh scatter based order of magnitude increase in distributed temperature and strain sensing by simple UV exposure of optical fibre," Scientific Reports, vol. 5, no. 1, p. 11177, 2015.

[41] P. S. Westbrook, K. S. Feder, R. M. Ortiz et al., "Kilometer length, low loss enhanced back scattering fiber for distributed sensing," in 2017 25th Optical Fiber Sensors Conference (OFS), Jeju, 2017.

[42] P. Lu, S. J. Mihailov, D. Coulas, H. Ding, and X. Bao, "Low loss random fiber gratings made with a fs-IR laser for distributed fiber sensing," Journal of Lightwave Technology, vol. 37, no. 18, pp. 4697-4702, 2019.

[43] X. Bao, D. P. Zhou, C. Baker, and L. Chen, "Recent development in the distributed fiber optic acoustic and ultrasonic detection," Journal of Lightwave Technology, vol. 35, no. 16, pp. 3256-3267, 2017.

[44] Y. Muanenda, C. J. Oton, S. Faralli, and F. Di Pasquale, "A cost-effective distributed acoustic sensor using a commercial off-the-shelf DFB laser and direct detection phase-OTDR," IEEE Photonics Journal, vol. 8, no. 1, pp. 1-10, 2016.

[45] M. Ren, P. Lu, L. Chen, and X. Bao, "Theoretical and experimental analysis of phase-OTDR based on polarization diversity detection," IEEE Photonics Technology Letters, vol. 28, no. 6, pp. 697-700, 2016.

[46] M. R. Fernández-Ruiz, L. Costa, and H. F. Martins, "Distributed acoustic sensing using chirped-pulse phase-sensitive OTDR technology," Sensors, vol. 19, no. 20, p. 4368, 2019.

[47] Y. Wang, P. Lu, S. Mihailov, L. Chen, and X. Bao, "Strain measurement range enhanced chirped pulse $\varphi$-OTDR for distributed static and dynamic strain measurement based on 
random fiber grating array," Optics Letters, vol. 45, no. 21, pp. 6110-6113, 2020.

[48] C. Spiegelberg, G. Jihong, H. Yongdan, Y. Kaneda, J. Shibin, and N. Peyghambarian, "Low-noise narrow-linewidth fiber laser at $1550 \mathrm{~nm}$ (June 2003)," Journal of Lightwave Technology, vol. 22, no. 1, pp. 57-62, 2004.

[49] H. Tsuchida, "Simple technique for improving the resolution of the delayed self-heterodyne method," Optics Letters, vol. 15, no. 11, pp. 640-642, 1990.

[50] L. Zhang, L. Chen, and X. Bao, "Unveiling delay-time-resolved phase noise statistics of narrow-linewidth laser via coherent optical time domain reflectometry," Optics Express, vol. 28, no. 5, pp. 6719-6733, 2020.

[51] B. Vanus, C. Baker, L. Chen, and X. Bao, "All-optical intensity fluctuation magnification using Kerr effect," Optics Express, vol. 28, no. 3, pp. 3789-3794, 2020.

[52] M. Ren, D. Zhou, L. Chen, and X. Bao, "Influence of finite extinction ratio on performance of phase-sensitive optical time-domain reflectometry," Optics Express, vol. 24, no. 12, pp. 13325-13333, 2016.

[53] C. Baker, B. Vanus, M. Wuilpart, L. Chen, and X. Bao, "Enhancement of optical pulse extinction-ratio using the nonlinear Kerr effect for phase-OTDR," Optics Express, vol. 24, no. 17, pp. 19424-19434, 2016.

[54] D. Iida, K. Toge, and T. Manabe, "Distributed measurement of acoustic vibration location with frequency multiplexed phaseOTDR," Optical Fiber Technology, vol. 36, pp. 19-25, 2017.

[55] Y. Muanenda, S. Faralli, C. J. Oton, and F. Di Pasquale, "Dynamic phase extraction in a modulated double-pulse $\phi$ OTDR sensor using a stable homodyne demodulation in direct detection," Optics Express, vol. 26, no. 2, pp. 687-701, 2018.

[56] S. Liehr, S. Münzenberger, and K. Krebber, "Wavelength-scanning coherent OTDR for dynamic high strain resolution sensing," Optics Express, vol. 26, no. 8, pp. 10573-10588, 2018.

[57] M. Froggatt and J. Moore, "High-spatial-resolution distributed strain measurement in optical fiber with Rayleigh scatter," Applied Optics, vol. 37, no. 10, pp. 1735-1740, 1998.

[58] J. Song, W. Li, P. Lu, Y. Xu, L. Chen, and X. Bao, "Long-range high spatial resolution distributed temperature and strain sensing based on optical frequency-domain reflectometry," IEEE Photonics Journal, vol. 6, no. 3, pp. 1-8, 2014.

[59] C. Baker, Y. Lu, J. Song, and X. Bao, "Incoherent optical frequency domain reflectometry based on a Kerr phase-interrogator," Optics Express, vol. 22, no. 13, pp. 15370-15375, 2014.

[60] C. Baker, L. Chen, and X. Bao, "The Kerr phase-interrogator: exploiting the nonlinear Kerr-effect for overcoming fundamental limitations in linear sensing approaches," Quantum Sensing and Nano Electronics and Photonics XV, 2018.

[61] S. Wang, X. Fan, Q. Liu, and Z. He, "Distributed fiber-optic vibration sensing based on phase extraction from time-gated digital OFDR," Optics Express, vol. 23, no. 26, pp. 3330133309, 2015.

[62] Q. Liu, X. Fan, and Z. He, "Time-gated digital optical frequency domain reflectometry with $1.6-\mathrm{m}$ spatial resolution over entire 110-km range," Optics Express, vol. 23, no. 20, pp. 25988-25995, 2015.

[63] D. P. Zhou, Z. Qin, W. Li, L. Chen, and X. Bao, "Distributed vibration sensing with time-resolved optical frequencydomain reflectometry," Optics Express, vol. 20, no. 12, pp. 13138-13145, 2012.
[64] D. P. Zhou, L. Chen, and X. Bao, "Distributed dynamic strain measurement using optical frequency-domain reflectometry," Applied Optics, vol. 55, no. 24, pp. 6735-6739, 2016.

[65] A. Masoudi and P. N. Trevor, "Contributed review: distributed optical fibre dynamic strain sensing," Review of scientific instruments, vol. 87, no. 1, article 011501, 2016.

[66] S. Zhao, J. Cui, L. Suo, Z. Wu, D. Zhou, and J. Tan, "Performance investigation of OFDR sensing system with a wide strain measurement range," Journal of Lightwave Technology, vol. 37, no. 15, pp. 3721-3727, 2019.

[67] W. Li, L. Chen, and X. Bao, "Compensation of temperature and strain coefficients due to local birefringence using optical frequency domain reflectometry," Optics Communications, vol. 311, pp. 26-32, 2013.

[68] Z. Ding, D. Yang, Y. Du et al., "Distributed strain and temperature discrimination using two types of fiber in OFDR," IEEE Photonics Journal, vol. 8, pp. 1-8, 2016.

[69] J. Zhang, H. Wu, H. Zheng et al., " $80 \mathrm{~km}$ fading free phasesensitive reflectometry based on multi-carrier NLFM pulse without distributed amplification," Journal of Lightwave Technology, vol. 37, no. 18, pp. 4748-4754, 2019.

[70] T. Zhu, Q. He, X. Xiao, and X. Bao, "Modulated pulses based distributed vibration sensing with high frequency response and spatial resolution," Optics Express, vol. 21, no. 3, pp. 2953-2963, 2013.

[71] Q. He, T. Zhu, J. Zhou, D. Diao, and X. Bao, "Frequency response enhancement by periodical nonuniform sampling in distributed sensing," IEEE Photonics Technology Letters, vol. 27, no. 20, pp. 2158-2161, 2015.

[72] J. Zhang, H. Zheng, T. Zhu et al., "Distributed fiber sparsewideband vibration sensing by sub-Nyquist additive random sampling," Optics Letters, vol. 43, no. 9, pp. 2022-2025, 2018.

[73] Y. Wang, P. Lu, S. Mihailov, L. Chen, and X. Bao, "Ultra-low frequency dynamic strain detection with laser frequency drifting compensation based on a random fiber grating array," Optics Letters, vol. 46, no. 4, pp. 789-792, 2021.

[74] K. Miah and D. K. Potter, "A review of hybrid fiber-optic distributed simultaneous vibration and temperature sensing technology and its geophysical applications," Sensors, vol. 17, no. 11, article 2511, 2017. 